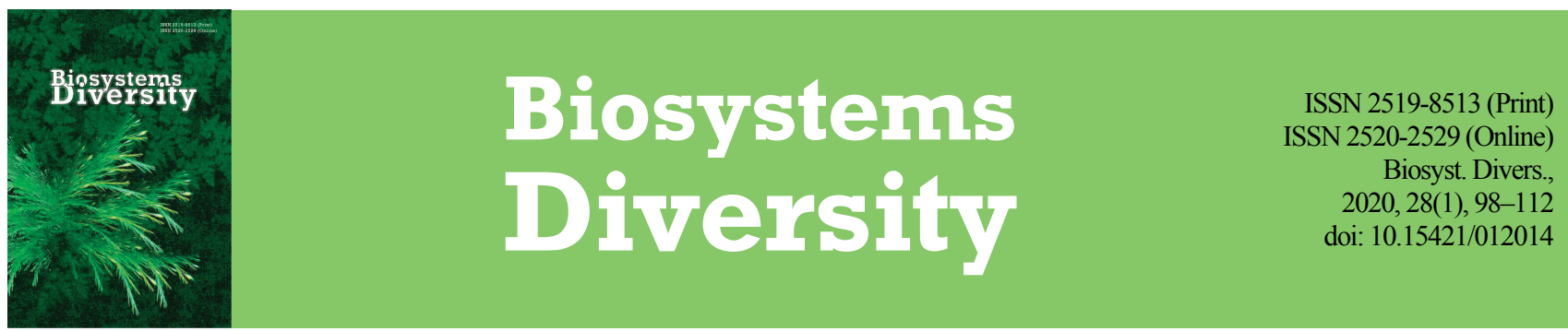

\title{
Ecological-climatic characteristics of the flora of a floodplain landscape in Southeastern Europe
}

\author{
B. A. Baranovski, L. A. Karmyzova, N. O. Roshchyna, I. A. Ivanko, O. G. Karas
}

\author{
Oles Honchar Dnipro National University, Dnipro, Ukraine
}

Article info

Received 02.02.2020

Received in revised form 27.02.2020

Accepted 28.02.2020

Oles Honchar Dnipro National University, Gagarin ave., 72,

Dnipro, 49010, Ukraine.

Tel.: + 38-095-779-99-94.

E-mail: boris.baranovski (a)kr.net

\section{Introduction}

Vascular plant flora is one of the most numerous and stable components which represents the state of ecosystems. The territories of European river floodplains are characterized by a high-level of biodiversity. They are represented by habitats with a high level of structural and functional dynamics (Schindler et al., 2016). Some of them can serve as reference sites with the best-preserved floral richness. One of these areas on the territory of the steppe zone of Eastern Europe is the floodplain landscape of the Samara River, a tributary of the Dnieper (Koshelev et al., 2020).

Today, the importance of forest ecosystems (especially in subarid regions) is of inestimable importance. They reduce the effects of global warming and create conditions for biodiversity conservation and recreation (Bussotti et al., 2015; Biurrun et al., 2016; Hossain et al., 2016; Colangelo et al., 2018). The development, sustainability and biodiversity of forest ecosystems, including those within floodplain habitats, depend mainly on climate and hydrological conditions (Mikac et al., 2 018; Stojanović et al., 2016). The only areas of forest growth in the steppe zone are the gullies (ravine forests) and floodplains (floodplain forests). The lack of atmospheric moisture is compensated by floodwaters. Tree species respond positively to floodwaters, even when floodplains are at their maximum (Heklau et al., 2019). The floodplain landscape is characterized by a richness of biotopes (forest, meadow, wetland), which create a variety of climatopical conditions not typical for steppe landscapes, especially in recent decades of global warming (Lavergne et al., 2010; Ortmann-Ajkai et al., 2018).

One of the significant woodlands of the steppe zone within Eastern Europe is the Samara Forest, which serves as the "southern outpost" of forest distribution in the steppe zone of Ukraine (Bel'gard, 1950). It is loca- ted downstream of the Samara River, a tributary of the Dnieper. On the territory of the steppe zone of Ukraine, this woodland is the only one of the remaining large floodplain forests of the middle river valleys. On the territory of the floodplain landscape of the Samara River, forest vegetation is represented by oak forests. The forests alternate with meadows, swamps, and water bodies (Brygadyrenko, 2015, 2016).

The diverse landscapes and rich flora of the Samara River area have attracted the attention of several well-known botanists. One of the species from the territory of of the Samara River area was described by Carl Linnaeus: Veronica incana L. (Elenevskij, 1978), the type stored in London. Detailed flora and vegetation surveys in this territory (including the floodplain landscape) were initiated at the end of the XIX century by Akinfiev (1908), the famous Ukrainian botanist. In the following years, the study of flora and vegetation was carried out by followers of the geobotanical scientific school of A. L. Belgard (Alekseyev et al., 1986, Tarasov et al., 1988; Tarasov, 2012; Baranovsky, 2016).

The flora of the Samara River area has historically been composed of different geographical elements and is distinguished by a large set of species and their ecomorphs (Belgard, 1950). The elements include a large number of rare plant species and a minimal number of adventive ones (Chervona knyha, 2010; Tarasov, 2012; Baranovsky, 2016). They are widely represented in all landscape elements of the Samara River area and especially in the floodplain. A variety of ecological conditions was developed in the forest area of the floodplain, which provides greater flora richness and diversity compared to transformed territories (Schindler et al., 2016).

In this regard, the task of our work was to compare the climatopes and ecofloristic diversity of the floodplain forest areas of the Samara River 
and anthropogenically transformed territories where the forest was destroyed. This is well illustrated by the A. L. Belgard Ecomorph System, which was the first vascular plant ecomorph system (Baranovsky et al., 2018). Finding out the specific values of phytodiversity in floodplain forest areas will help initiate measures for their protection and restoration.

\section{Materials and methods}

The survey subject was the microclimatic features and floodplain vascular flora of the Samara River in its downstream area (between Kocherechki village and Volnoye village). Coordinates: Volnoye: $48.7281^{\circ} \mathrm{N}, 35.2931^{\circ} \mathrm{E}$; Kocherechki $48.6370^{\circ} \mathrm{N}, 35.6900^{\circ} \mathrm{E}$; IvanoMikhaylovka $48.7736^{\circ} \mathrm{N}, 35.3750^{\circ} \mathrm{E}$; Cherkasskoye $48.6786^{\circ} \mathrm{N}$, $35.3764^{\circ} \mathrm{E}$ (Fig. 1). One of the climate features in the Samara River area, which is located within the steppe Atlantic-continental climate type, is the high temperatures of the warm period with reduced precipitation and significant air dryness. The average annual air temperature is $8.1^{\circ} \mathrm{C}$, and the average annual precipitation is $545 \mathrm{~mm}$ (Gorb \& Duk, 2006). Average annual amplitude of air temperature is $26.8^{\circ} \mathrm{C}$. The average temperature and humidity (Table 1) of this region (Hubynykha weather forecast) show the most intense temperature rise in the period from March to April $\left(8.8^{\circ} \mathrm{C}\right)$ and the most intense drop in temperature from September to October $\left(6.9^{\circ} \mathrm{C}\right)$.

The scientific basis for the survey of climatopes in native forest biogeocenoses was the doctrine of the forests' influence on environmental change (the doctrine of forest pertinence) of Vysotsky (1950). Climate research was conducted according to widely accepted methods. A set of standard methods of hydrometeorological observations was carried out at the test sites, and the gradient climate stand proposed by Gritsan (2000) was used, which allows meteorological elements to be measured at any level from the soil surface and tree crowns (Grytsan et al., 2005) The hydrological features of the of the middle river floodplains in the steppe zone are characterized by short-term flooding (Ramenskiy, 1971). The territory of the Samara River floodplain is flooded during high water for a period of about one week (Belgard, 1950). This determines mainly sufficient moisture for oak forests under short-term flooding conditions.

\section{Table 1}

Climatic indicators of the Samara River area (Hubynykha)

\begin{tabular}{|c|c|c|c|c|c|c|c|c|c|c|c|c|}
\hline Month & $\mathrm{I}$ & II & III & IV & $\mathrm{V}$ & VI & VII & VIII & IX & $\mathrm{X}$ & $\mathrm{XI}$ & XII \\
\hline Average monthly air temperature ${ }^{\circ} \mathrm{C}^{*}$ & -6.1 & -4.7 & 0.4 & 9.2 & 15.9 & 19.2 & 20.7 & 20.0 & 14.8 & 7.9 & 2.0 & -2.6 \\
\hline Average monthly relative air humidity $\%$ & 89 & 88 & 84 & 68 & 60 & 61 & 61 & 60 & 65 & 77 & 87 & 89 \\
\hline Precipitation norm, mm & 48 & 34 & 31 & 40 & 46 & 69 & 58 & 43 & 41 & 36 & 44 & 55 \\
\hline
\end{tabular}

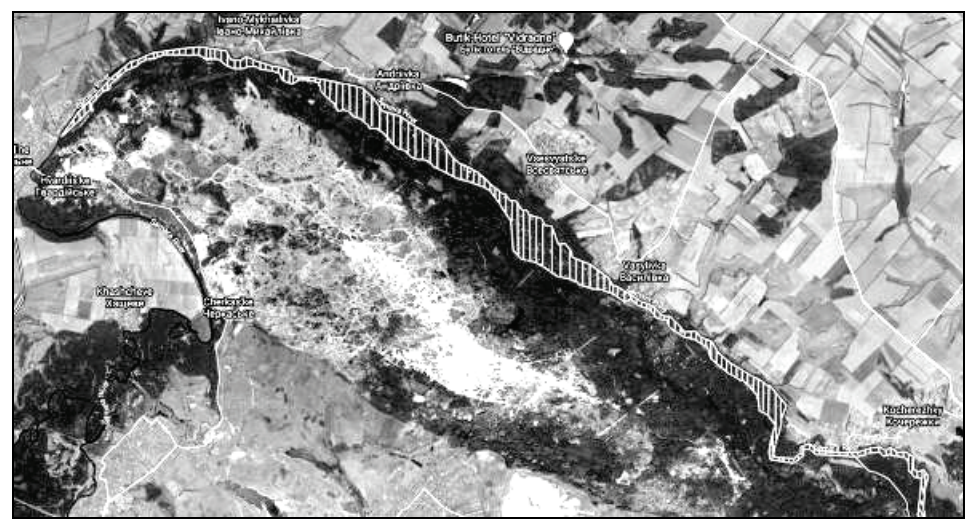

Fig. 1. Diagrammatic map of the downstream territory of the Samara River area (hatching indicates the floodplain territory)

The vascular plant flora survey was conducted from 1998 to 2019. To compile a complete flora list, materials from the herbarium collections of Dnipropetrovsk University and the National Herbarium of the Institute of Botany, National Academy of Sciences of Ukraine were used. 1300 herbarium samples were collected. The flora of different floodplain biotopes and different types of water bodies was surveyed (Roshchyna \& Baranovski, 2019). The definition of vascular plant species was carried out according to the following identification manuals and "Flora" (Flora of the USSR, 1935-1963; Prokudin \& Dobrochaeva, 1987). Plant species names were given according to the nomenclature publication adopted in Ukraine (Mosyakin \& Fedorochuk, 1999).

Bioecomorphic flora analysis was carried out according to the ecomorph system of Belgard (1950). For ecomorphic characterization of species, we used existing publications (Belgard, 1950; Baranovski, 2000; Tarasov, 2012), consultations with leading experts of the Institute of Botany of National Academy of Sciences of Ukraine, as well as our presonal data (Baranovsky et al., 2017, 2018).

\section{Results}

The greatest differences in meteorological elements were observed between the floodplain and steppe landscapes in summer during the period of the greatest development of crown leaves. The weakened turbulent air exchange reduces the average daily temperature by $0.5-2.0{ }^{\circ} \mathrm{C}$ and increases the average daily relative humidity by $10-16 \%$. The maximum temperature of the soil surface is reduced by $7-15{ }^{\circ} \mathrm{C}$ in comparison with the values of steppe landscape. With variable cloud cover, amplitude of temperature fluctuations of the soil surface in forest areas on average is up to $7.0^{\circ} \mathrm{C}$, and in conditions of the steppe landscape the amplitude is more than $20^{\circ} \mathrm{C}$.

Forested areas have inherent climate features in a number of different biotopes: riverbeds, lakes, swamps, forest margins, and glades. Surveys of climatopes have revealed that light intensity and energy supply in the field of PAR (photosynthetically active radiation) increases in the following sequence of biotopes: oak forest $\rightarrow$ forest margin $\rightarrow$ woodland water body. Light intensity under the canopy of oak forest during the light period of the day is reduced by an average of $95.2 \%$ in comparison with nonwooded areas. Significant reflectivity of the surface of a water body provides additional solar radiation in the FAW range of the lower part of the crowns and undergrowth. Amplitude of daily temperature fluctuations in the forest glade biotope is 1.4 times greater than in the water body and forest biotopes.

All these factors impact significantly on the ecosystem functioning. They provide the basis of the floral diversity of floodplain biogeocenoses. Floral diversity of the biogeocenoses is incomparably richer than the rest of the territory. The flora of vascular plants of the Samara floodplain is very diverse both in systematic and ecological terms. A wide range of different ecomorphs are represented here (Table 2).

The vascular plant species list of the floodplain habitats in the Samara forest includes 728 species. They belong to 4 divisions, 5 classes, 108 families, 429 genera (Table 2). Of these, 148 species are rare, which are included in the European Red List (2 species), the Red Book of Ukraine (24 species) and the regional Red List of the Dnipropetrovsk region (148 species) (Chervona knyha..., 2010). 
Table 2

Flora of the floodplain landscape in the Samara River downstream

\begin{tabular}{|c|c|c|c|c|c|c|c|c|c|}
\hline Family Views & $\begin{array}{l}\text { Clima- } \\
\text { morphs }\end{array}$ & $\begin{array}{l}\text { Thermo- } \\
\text { morphs }\end{array}$ & $\begin{array}{l}\text { Helio- } \\
\text { morphs }\end{array}$ & $\begin{array}{l}\text { Tropho- } \\
\text { morphs }\end{array}$ & $\begin{array}{l}\text { Hygro- } \\
\text { morphs }\end{array}$ & $\begin{array}{l}\text { Ceno- } \\
\text { morphs }\end{array}$ & $\begin{array}{l}\text { The rare } \\
\text { flora frac- } \\
\text { tion, adven- } \\
\text { tive status }\end{array}$ & $\begin{array}{l}\text { Types of } \\
\text { forested } \\
\text { areas }\end{array}$ & $\begin{array}{c}\text { Types of } \\
\text { non-forested } \\
\text { areas }\end{array}$ \\
\hline 1 & 2 & 3 & 4 & 5 & 6 & 7 & 8 & 9 & 10 \\
\hline \multicolumn{10}{|l|}{$\begin{array}{l}\text { Divisio Equisetophyta } \\
\text { Equisetaceae }\end{array}$} \\
\hline Equisetum arvense L. & G & MsT & $\mathrm{ScHe}$ & $\mathrm{MsTr}$ & $\mathrm{HgMs}$ & RuSilPr & - & + & + \\
\hline E. fluviatile $\mathrm{L}$. & $\mathrm{HKr}$ & MsT & $\mathrm{ScHe}$ & MsTr & $\mathrm{HelHg}$ & AqPal & RLD & + & + \\
\hline E. hyemale $\mathrm{L}$. & $\mathrm{Ch}$ & MsT & $\mathrm{ScHe}$ & $\mathrm{MsTr}$ & $\mathrm{HgMs}$ & PrSil & RLD & + & - \\
\hline E. pratense Ehrh. & $\mathrm{G}$ & MsT & $\mathrm{ScHe}$ & MsTr & HgMs & $\operatorname{Pr}$ & - & - & + \\
\hline E. ramosissimum Desf. & $\mathrm{G}$ & MsT & $\mathrm{ScHe}$ & $\mathrm{MsOgTr}$ & Ms & PrPs & - & - & + \\
\hline $\begin{array}{l}\text { E. sylvaticum L. } \\
\text { Divisio Polypodiophyta }\end{array}$ & G & MsT & $\mathrm{Sc}$ & $\mathrm{MsTr}$ & $\mathrm{HgMs}$ & Sil & RLD & + & - \\
\hline $\begin{array}{c}\text { Aspleniaceae } \\
\text { Asplenium trichomanes } \mathrm{L} \text {. } \\
\text { Athyriaceae }\end{array}$ & $\mathrm{HKr}$ & MsT & $\mathrm{HeSc}$ & MsTr & Ms & SilPt & RLD & + & - \\
\hline Athyrium filix-femina (L.) Roth & $\mathrm{HKr}$ & $\operatorname{MgT}$ & Sc & MsTr & $\mathrm{HgMs}$ & Sil & RLD & + & - \\
\hline $\begin{array}{l}\text { Cystopteris fragilis Bernh. } \\
\text { Dennstaedtiaceae }\end{array}$ & $\mathrm{HKr}$ & $\mathrm{MgT}$ & $\mathrm{HeSc}$ & $\mathrm{MsOgTr}$ & Ms & PsSil & RLD & + & - \\
\hline $\begin{array}{l}\text { Pteridium aquilinum (L.) Kuhn } \\
\text { Dryopteriaceae }\end{array}$ & G & MT & $\mathrm{HeSc}$ & OgMsTr & Ms & Sil & RLD & + & - \\
\hline Dryopteris carthusiana H. P. Fuchs. & $\mathrm{HKr}$ & MsT & $\mathrm{ScHe}$ & MsTr & Ms & Sil & RLD & + & - \\
\hline D. cristata $(\mathrm{L}$.$) A. Grey$ & $\mathrm{HKr}$ & MsT & $\mathrm{HeSc}$ & OgMsTr & $\mathrm{MsHg}$ & Sil & RLD & + & - \\
\hline D. filix-mas (L.) Schott. & $\mathrm{HKr}$ & MsT & $\mathrm{HeSc}$ & OgMsTr & Ms & Sil & RLD & + & - \\
\hline $\begin{array}{r}\text { Polystichum aculeatum (L.) Roth } \\
\text { Onocleaceae }\end{array}$ & $\mathrm{HKr}$ & $\mathrm{MgT}$ & $\mathrm{Sc}$ & OgMsTr & $\mathrm{HgMs}$ & PtSil & RLD & + & - \\
\hline $\begin{array}{r}\text { Matteuccia struthiopteris (L.) Tod. } \\
\text { Ophioglossaceae }\end{array}$ & $\mathrm{HKr}$ & MsT & $\mathrm{Sc}$ & MsTr & HgMs & Sil & RLD & + & - \\
\hline $\begin{array}{l}\text { Botrychium lunaria (L.) Sw. } \\
\text { Polypodiaceae }\end{array}$ & G & $\mathrm{MgT}$ & $\mathrm{He}$ & $\mathrm{MsTr}$ & Ms & $\operatorname{Pr}$ & RBU, RLD & + & - \\
\hline $\begin{array}{l}\text { Polypodium vulgare L. } \\
\text { Salviniaceae }\end{array}$ & $\mathrm{HKr}$ & MT & $\mathrm{HeSc}$ & $\mathrm{MsTr}$ & Ms & SilPt & RLD & + & - \\
\hline $\begin{array}{l}\text { Salvinia natans (L.) All. } \\
\text { Thelypteridaceae }\end{array}$ & $\mathrm{T}$ & MsT & $\mathrm{ScHe}$ & $\mathrm{MsTr}$ & $\mathrm{Pl}$ & $\mathrm{Aq}$ & RBU, RLD & + & - \\
\hline $\begin{array}{l}\text { Thelypteris palustris Schott } \\
\text { Divisio Pinophyta } \\
\text { Pinaceae }\end{array}$ & G & MT & $\mathrm{HeSc}$ & $\mathrm{MsTr}$ & $\mathrm{Hg}$ & SilPal & RLD & + & - \\
\hline $\begin{array}{l}\text { Pinus sylvestris L. } \\
\text { Divisio Magnoliophyta } \\
\text { Class Liliopsida } \\
\text { Alismataceae }\end{array}$ & $\mathrm{Ph}$ & MsT & $\mathrm{ScHe}$ & OgMsTr & Ms & Sil & - & + & - \\
\hline Alisma gramineum Ley. & $\mathrm{HKr}$ & $\operatorname{MgT}$ & $\mathrm{He}$ & $\operatorname{Ms} \operatorname{Tr}$ & $\mathrm{HelHg}$ & PalAq & - & - & + \\
\hline A. lanceolatum With. & $\mathrm{HKr}$ & $\mathrm{MgT}$ & $\mathrm{He}$ & $\mathrm{MgTr}$ & $\mathrm{HelHg}$ & PalAq & - & + & + \\
\hline A. plantago-aquatica $\mathrm{L}$. & $\mathrm{HKr}$ & MT & $\mathrm{ScHe}$ & MsTr & $\mathrm{HgHel}$ & PalAq & - & + & + \\
\hline $\begin{array}{l}\text { Sagittaria sagittifolia } \mathrm{L} \text {. } \\
\text { Alliaceae }\end{array}$ & $\mathrm{HKr}$ & MsT & $\mathrm{ScHe}$ & $\mathrm{MsTr}$ & $\mathrm{HgHy}$ & PalAq & - & + & + \\
\hline Allium angulosum $\mathrm{L}$. & G & MsT & $\mathrm{ScHe}$ & MsTr & $\mathrm{HgMs}$ & $\operatorname{Pr}$ & - & + & + \\
\hline A. guttatum Stev. & $\mathrm{G}$ & MsT & $\mathrm{He}$ & $\mathrm{OgMgTr}$ & $\mathrm{OgX}$ & PsSt & RLD & - & + \\
\hline A. oleraceum $\mathrm{L}$. & G & MsT & $\mathrm{ScHe}$ & MsOgTr & $\mathrm{XMs}$ & RuStPrSil & - & + & + \\
\hline $\begin{array}{l}\text { A. waldschteinii G.Don fil. } \\
\text { Araceae }\end{array}$ & $G$ & $M g T$ & $\mathrm{He}$ & MsTr & $\mathrm{XMs}$ & St & - & + & + \\
\hline $\begin{array}{l}\text { Acorus calamus } \mathrm{L} \text {. Butomaceae } \\
\end{array}$ & $\mathrm{HKr}$ & $\mathrm{MgT}$ & $\mathrm{He}$ & $\mathrm{MsTr}$ & $\mathrm{HgHel}$ & PalAq & RLD, Adv & + & + \\
\hline $\begin{array}{l}\text { Butomus umbellatus } \mathrm{L} \text {. } \\
\text { Convallariaceae }\end{array}$ & $\mathrm{HKr}$ & $\mathrm{MgT}$ & $\mathrm{He}$ & $\mathrm{MsTr}$ & $\mathrm{HgHel}$ & PalAq & - & + & + \\
\hline Convallaria majalis $\mathrm{L}$. & $\mathrm{G}$ & $\mathrm{MgT}$ & $\mathrm{HeSc}$ & MsTr & $\mathrm{HgMs}$ & Sil & RLD & + & - \\
\hline Polygonatum multiflorum (L.) All. & G & $\mathrm{MgT}$ & $\mathrm{Sc}$ & $\operatorname{MgTr}$ & Ms & Sil & - & + & - \\
\hline $\begin{array}{l}\text { P. odoratum (Mill.) Druce } \\
\text { Cyperaceae }\end{array}$ & G & MsT & $\mathrm{ScHe}$ & OgTr & Ms & Sil & RLD & + & - \\
\hline Bolboschoenus compactus (Hoffm.) Drob. & $\mathrm{HKr}$ & $\mathrm{MgT}$ & $\mathrm{He}$ & AlkMsTr & $\mathrm{HelHg}$ & PrPal & - & + & + \\
\hline B. maritimus (L.) Palla & $\mathrm{HKr}$ & $\mathrm{MgT}$ & $\mathrm{He}$ & AlkMgTr & $\mathrm{HelHg}$ & AqPal & - & + & + \\
\hline Carex acuta $\mathrm{L}$. & $\mathrm{HKr}$ & $\mathrm{MgT}$ & $\mathrm{He}$ & MsTr & $\mathrm{HelHg}$ & AqPal & - & + & + \\
\hline C. acutiformis Ehrh. & $\mathrm{HKr}$ & $\mathrm{MgT}$ & $\mathrm{ScHe}$ & MsTr & $\mathrm{Hg}$ & SilPal & - & + & + \\
\hline C. bohemica Schreb. & $\mathrm{HKr}$ & MsT & $\mathrm{He}$ & OgTr & $\mathrm{MsHg}$ & PrPs & RBU, RLD & + & + \\
\hline C. bueckii Wimm. & $\mathrm{HKr}$ & MsT & $\mathrm{He}$ & AlkMgTr & $\mathrm{HgMs}$ & PalPr & RBU, RLD & + & + \\
\hline C. caryophyllea Latourr. & $\mathrm{HKr}$ & MsT & $\mathrm{He}$ & $\operatorname{Og} \operatorname{Tr}$ & Ms & PrPs & - & + & + \\
\hline C. caespitosa $\mathrm{L}$. & $\mathrm{HKr}$ & $\mathrm{MgT}$ & $\mathrm{ScHe}$ & MsTr & $\mathrm{MsHg}$ & PrPal & - & + & - \\
\hline C. distans $\mathrm{L}$. & $\mathrm{HKr}$ & $\operatorname{MgT}$ & $\mathrm{He}$ & AlkMgTr & $\mathrm{HgMs}$ & PrHal & - & - & + \\
\hline C. disticha Huds. & $\mathrm{HKr}$ & $\mathrm{MgT}$ & $\mathrm{He}$ & $\operatorname{MsTr}$ & HsMs & PalPr & - & + & + \\
\hline C. elata All. & $\mathrm{HKr}$ & $\mathrm{MgT}$ & HSc & OgTr & $\mathrm{MsHg}$ & SilPal & RLD & + & - \\
\hline C. hirta $\mathrm{L}$. & $\mathrm{HKr}$ & $\mathrm{MgT}$ & $\mathrm{ScHe}$ & OgMsTr & $\mathrm{HgMs}$ & SilPr & - & + & + \\
\hline C. lasiocarpa Ehrh. & $\mathrm{HKr}$ & MsT & $\mathrm{ScHe}$ & $\mathrm{Og} \operatorname{Tr}$ & $\mathrm{MsHg}$ & SilPal & RBU, RLD & + & - \\
\hline C. lachenalii Schuhr & $\mathrm{HKr}$ & $\mathrm{MgT}$ & $\mathrm{ScHe}$ & MsTr & $\mathrm{Ms}$ & SilPr & - & + & - \\
\hline
\end{tabular}




\begin{tabular}{|c|c|c|c|c|c|c|c|c|c|}
\hline 1 & 2 & 3 & 4 & 5 & 6 & 7 & 8 & 9 & 10 \\
\hline C. limosa L. & $\mathrm{HKr}$ & MsT & $\mathrm{He}$ & MsTr & $\mathrm{MsHg}$ & Pal & - & + & + \\
\hline C. melanostachya Bieb. ex Willd. & $\mathrm{HKr}$ & MsT & $\mathrm{ScHe}$ & AlkMgTr & Ms & PalPrSil & - & + & + \\
\hline C. michelii Host & $\mathrm{HKr}$ & $\operatorname{MgT}$ & $\mathrm{HeSc}$ & MsTr & XMs & StSil & - & + & - \\
\hline C. muricata L. & $\mathrm{HKr}$ & MgT & $\mathrm{ScHe}$ & $\operatorname{MgTr}$ & Ms & Sil & - & + & - \\
\hline C. otrubae Podp. & $\mathrm{HKr}$ & MsT & $\mathrm{ScHe}$ & $\operatorname{MgTr}$ & HgMs & HalSilPr & - & + & + \\
\hline C. praecox Schreb. & $\mathrm{HKr}$ & MgT & $\mathrm{He}$ & $\operatorname{MgTr}$ & XMs & StPr & - & + & + \\
\hline C. pseudocyperus L. & $\mathrm{HKr}$ & $\mathrm{MgT}$ & $\mathrm{HeSc}$ & OgMsTr & $\mathrm{Hg}$ & SilPal & - & + & - \\
\hline C. rhisina Blitt ex Lindbl. & $\mathrm{HKr}$ & MsT & $\mathrm{Sc}$ & MsTr & Ms & Sil & RLD & + & - \\
\hline C. riparia Curtis & $\mathrm{HKr}$ & $\mathrm{MgT}$ & $\mathrm{He}$ & MsTr & $\mathrm{Hg}$ & Pal & - & + & + \\
\hline C. spicata Huds. & $\mathrm{HKr}$ & MgT & $\mathrm{HeSc}$ & $\operatorname{MgTr}$ & $\mathrm{XMs}$ & PrSil & - & + & - \\
\hline C. stenophylla Wahlenb. & $\mathrm{HKr}$ & MgT & $\mathrm{He}$ & AlkMgTr & $\mathrm{MsX}$ & PrSt & - & - & + \\
\hline C. vesicaria $\mathrm{L}$. & $\mathrm{HKr}$ & MsT & $\mathrm{He}$ & MsTr & $\mathrm{MsHg}$ & PrPal & - & + & + \\
\hline C. vulpina $\mathrm{L}$. & $\mathrm{HKr}$ & $\mathrm{MgT}$ & $\mathrm{He}$ & MsTr & $\mathrm{MsHg}$ & PalPr & - & + & + \\
\hline Cyperus fuscus $\mathrm{L}$. & $\mathrm{T}$ & $\mathrm{MgT}$ & $\mathrm{He}$ & OgMsTr & $\mathrm{Hg}$ & PsPal & - & + & + \\
\hline Eleocharis acicularis (L.) Roem. et Schult. & $\mathrm{HKr}$ & MgT & $\mathrm{ScHe}$ & MsTr & $\mathrm{HelHg}$ & PrPal & RLD & + & - \\
\hline E. mitracarpa Steud. & $\mathrm{HKr}$ & MgT & $\mathrm{He}$ & $\operatorname{MgTr}$ & $\mathrm{Hg}$ & PrPal & - & + & + \\
\hline E. palustris (L.) Roem. et Schult. & $\mathrm{HKr}$ & $\mathrm{MgT}$ & $\mathrm{He}$ & $\operatorname{MgTr}$ & $\mathrm{Hg}$ & PrPal & - & + & + \\
\hline Pycreus flavescens Reichenb. & $\mathrm{T}$ & $\mathrm{MgT}$ & $\mathrm{He}$ & MsOgTr & $\mathrm{Hg}$ & PsPal & - & + & + \\
\hline Scirpoides holoschoenus (L.) Sojak. & $\mathrm{G}$ & $\mathrm{MgT}$ & $\mathrm{He}$ & $\mathrm{OgTr}$ & $\mathrm{HgMs}$ & PrPs & - & + & + \\
\hline Scirpus lacustris L. & $\mathrm{HKr}$ & MgT & $\mathrm{He}$ & MsTr & $\mathrm{HelHg}$ & AqPal & - & + & + \\
\hline S. supinus (L.) Palla & $\mathrm{T}$ & $\mathrm{MgT}$ & $\mathrm{ScHe}$ & MsTr & $\mathrm{MsHg}$ & PrPal & RLD & + & + \\
\hline S. sylvaticus $\mathrm{L}$. & $\mathrm{HKr}$ & $\mathrm{MgT}$ & $\mathrm{HeSc}$ & MsTr & $\mathrm{Hg}$ & SilPal & - & + & - \\
\hline \multicolumn{9}{|l|}{ Hyacintaceae } & + \\
\hline Ornithogalum bouscheanum (Kunth) Aschers. & $\mathrm{G}$ & $\operatorname{MgT}$ & $\mathrm{HeSc}$ & MsTr & Ms & PrSil & RBU, RLD & + & - \\
\hline O. fimbriatum Willd. & $\mathrm{G}$ & $\mathrm{MgT}$ & $\mathrm{ScHe}$ & MsTr & Ms & Sil & RLD & + & - \\
\hline O. kochii Parl. & $\mathrm{G}$ & MgT & $\mathrm{He}$ & MsTr & MsX & SilPtSt & RLD & + & + \\
\hline Scilla bifolia $\mathrm{L}$. & $\mathrm{G}$ & MgT & $\mathrm{ScHe}$ & MsTr & $\mathrm{XMs}$ & StSil & RLD & + & - \\
\hline S. sibirica Haw. & $\mathrm{G}$ & MgT & $\mathrm{HeSc}$ & $\operatorname{MgTr}$ & Ms & Sil & RLD & + & - \\
\hline \multicolumn{10}{|l|}{ Hydrocharitaceae } \\
\hline Elodea canadensis Michx. & $\mathrm{Hd}$ & EuT & $\mathrm{HeSc}$ & OgMsTr & Hy & $\mathrm{RuAq}$ & Adv & + & + \\
\hline Hydrocharis morsus-ranae $\mathrm{L}$. & $\mathrm{Hd}$ & MsT & $\mathrm{ScHe}$ & MsTr & $\mathrm{Pl}$ & $\mathrm{Aq}$ & - & + & + \\
\hline Stratiotes aloides L. & $\mathrm{Hd}$ & MsT & $\mathrm{ScHe}$ & $\mathrm{MsTr}$ & $\mathrm{Pl}$ & $\mathrm{Aq}$ & RLD & + & + \\
\hline \multicolumn{9}{|l|}{ Iridaceae } & + \\
\hline Crocus reticulatus Stev. ex Adam. & $\mathrm{G}$ & MgT & $\mathrm{He}$ & AlkMsTr & MsX & PrSt & RBU, RLD & - & + \\
\hline Gladiolus tenuis Bieb. & $\mathrm{G}$ & MgT & $\mathrm{ScHe}$ & MsTr & $\mathrm{XMs}$ & $\operatorname{Pr}$ & RBU, RLD & - & + \\
\hline Iris halophila Pall. & $\mathrm{HKr}$ & MsT & $\mathrm{He}$ & AlkMsTr & $\mathrm{XMs}$ & HalPr & RLD & - & + \\
\hline I. pseudacorus L. & $\mathrm{G}$ & $\mathrm{MgT}$ & $\mathrm{He}$ & MsTr & $\mathrm{Hg}$ & $\mathrm{Pal}$ & - & + & + \\
\hline \multicolumn{10}{|l|}{ Juncaceae } \\
\hline Juncus articulatus L. & $\mathrm{HKr}$ & MsT & $\mathrm{He}$ & OgMsTr & $\mathrm{MsHg}$ & PalPr & - & + & + \\
\hline J. atratus Krook. & $\mathrm{HKr}$ & MsT & $\mathrm{He}$ & $\operatorname{MgTr}$ & $\mathrm{HgMs}$ & $\operatorname{Pr}$ & - & + & + \\
\hline J. bufonius L. & $\mathrm{T}$ & MsT & $\mathrm{ScHe}$ & OgMsTr & $\mathrm{MsHg}$ & PsPr & - & + & + \\
\hline J. compressus Jacq. & $\mathrm{HKr}$ & $\mathrm{MgT}$ & $\mathrm{He}$ & $\operatorname{MgTr}$ & $\mathrm{MsHg}$ & PalPr & - & + & + \\
\hline J. effusus L. & $\mathrm{Hel}$ & MgT & $\mathrm{He}$ & MsTr & $\mathrm{Hg}$ & $\mathrm{Pal}$ & - & + & + \\
\hline J. gerardii Loisel. & $\mathrm{HKr}$ & MgT & $\mathrm{He}$ & AlkMsTr & $\mathrm{HgMs}$ & HalPr & - & + & + \\
\hline J. inflexus L. & $\mathrm{HKr}$ & MgT & $\mathrm{He}$ & MsTr & $\mathrm{MsHg}$ & $\operatorname{Pr}$ & - & - & + \\
\hline J. tenageia Ehrh. ex L.fil. & $\mathrm{T}$ & MgT & $\mathrm{He}$ & $\operatorname{Og} \operatorname{Tr}$ & $\mathrm{MsHg}$ & PrPs & - & + & + \\
\hline Lusula multiflora (Ehrh.) Lej. & $\mathrm{HKr}$ & MgT & $\mathrm{ScHe}$ & MsTr & Ms & SilPr & RLD & + & - \\
\hline L. pallidula Krischner & $\mathrm{HKr}$ & MgT & $\mathrm{ScHe}$ & MsTr & Ms & SilPr & - & + & - \\
\hline \multicolumn{10}{|l|}{ Juncaginaceae } \\
\hline Triglochin maritimum L. & $\mathrm{HKr}$ & MgT & $\mathrm{He}$ & AlkTr & $\mathrm{MsHg}$ & PalHalPr & - & + & + \\
\hline T. palustre $\mathrm{L}$. & $\mathrm{HKr}$ & MgT & $\mathrm{He}$ & $\operatorname{MgTr}$ & $\mathrm{MsHg}$ & HalPalPr & - & + & + \\
\hline \multicolumn{10}{|l|}{ Lemnaceae } \\
\hline Lemna gibba $\mathrm{L}$. & $\mathrm{Hel}$ & EuT & $\mathrm{ScHe}$ & $\operatorname{MsTr}$ & $\mathrm{Pl}$ & $\mathrm{Aq}$ & - & + & + \\
\hline L. minor L. & Hel & EuT & $\mathrm{ScHe}$ & MsTr & $\mathrm{Pl}$ & $\mathrm{Aq}$ & - & + & + \\
\hline L. trisulca L. & Hel & MsT & $\mathrm{HeSc}$ & MsTr & Hy & $\mathrm{Aq}$ & - & + & + \\
\hline Spirodela polirrhyza (L.) Schleid. & Hel & EuT & $\mathrm{He}$ & MsTr & $\mathrm{Pl}$ & $\mathrm{Aq}$ & - & + & + \\
\hline $\begin{array}{c}\text { Wolffia arrhiza }(\text { L.) Horkel ex Wimm. } \\
\text { Liliaceae }\end{array}$ & Hel & EuT & $\mathrm{He}$ & MsTr & $\mathrm{Pl}$ & $\mathrm{Aq}$ & RLD & + & + \\
\hline Fritillaria meleagroides Patrin ex Schult. et Schult. f. & $\mathrm{G}$ & MsT & $\mathrm{He}$ & AlkMgTr & Ms & $\operatorname{Pr}$ & RBU, RLD & + & + \\
\hline Fritillaria ruthenica WiXtr. & $\mathrm{G}$ & MsT & $\mathrm{ScHe}$ & MsTr & $\mathrm{XMs}$ & Sil & RBU, RLD & + & - \\
\hline Gagea bulbifera (Pall.) Salisb. & $\mathrm{G}$ & MgT & $\mathrm{He}$ & MsTr & MsX & PtSt & - & + & + \\
\hline G. erubscens (Bess.) Schult.et Schult.fil. & $\mathrm{G}$ & MsT & $\mathrm{ScHe}$ & $\operatorname{MgTr}$ & Ms & RuSil & - & + & + \\
\hline G. lutea (L.) Ker-Gawl. & $\mathrm{G}$ & $\mathrm{MgT}$ & $\mathrm{HeSc}$ & MsTr & Ms & Sil & RLD & + & - \\
\hline Tulipa quercetorum Klokov et Zoz & $\mathrm{G}$ & MsT & $\mathrm{HeSc}$ & $\operatorname{MgTr}$ & Ms & StSil & RBU, RLD & + & + \\
\hline Najadaceae & & & & & & & & & \\
\hline Caulinia minor (All.) Coss.et Germ. & $\mathrm{T}$ & EuT & $\mathrm{HeSc}$ & MsTr & Hy & $\mathrm{Aq}$ & RLD & + & + \\
\hline Najas marina $\mathrm{L}$. & $\mathrm{T}$ & $\mathrm{MgT}$ & $\mathrm{HeSc}$ & MsTr & Hy & $\mathrm{Aq}$ & - & + & + \\
\hline Orchidaceae & & & & & & & & & \\
\hline Dactylorhisa incarnata L. & G & MsT & $\mathrm{He}$ & $\operatorname{MgTr}$ & $\mathrm{MsHg}$ & PrPal & RBU, RLD & + & + \\
\hline Anacamptis morio L. & $\mathrm{G}$ & MsT & $\mathrm{He}$ & $\operatorname{MgTr}$ & $\mathrm{XMs}$ & $\operatorname{Pr}$ & RBU, RLD & + & + \\
\hline A. palustris (Jacq.) R.M. Bataman & $\mathrm{G}$ & $\mathrm{MgT}$ & $\mathrm{He}$ & AlkMsTr & $\mathrm{MsHg}$ & PrPal & RBU, RLD & - & + \\
\hline Epipactis helleborine (L.) Crantz & $\mathrm{G}$ & MgT & $\mathrm{ScHe}$ & MsTr & Ms & PrSil & RBU, RLD & + & - \\
\hline E. palustris (L.) Crantz & $\mathrm{G}$ & MgT & $\mathrm{ScHe}$ & OgMsTr & $\mathrm{MsHg}$ & PalPr & RBU, RLD & + & + \\
\hline Liparis loeselii (L.) Rich. & $\mathrm{G}$ & MgT & $\mathrm{He}$ & OgMsTr & $\mathrm{MsHg}$ & PrPal & RBU, RLD & + & + \\
\hline Listera ovata (L.) R. Borbas & $\mathrm{G}$ & MsT & $\mathrm{HeSc}$ & MsTr & Ms & Sil & RBU, RLD & + & - \\
\hline
\end{tabular}




\begin{tabular}{|c|c|c|c|c|c|c|c|c|c|}
\hline 1 & 2 & 3 & 4 & 5 & 6 & 7 & 8 & 9 & 10 \\
\hline Neottia nidus-avis (L.) Rich. & $\mathrm{HKr}$ & MsT & $\mathrm{Sc}$ & MsTr & Ms & Sil & RBU, RLD & + & - \\
\hline Orchis militaris L. & $\mathrm{G}$ & $\mathrm{MgT}$ & $\mathrm{HeSc}$ & MsTr & Ms & SilPr & RBU, RLD & - & + \\
\hline O. ustulata L. & $\mathrm{G}$ & $\mathrm{MgT}$ & $\mathrm{ScHe}$ & $\operatorname{MgTr}$ & Ms & SilPr & RBU, RLD & + & + \\
\hline Platanthera bifolia (L.) Rich. & $\mathrm{G}$ & $\mathrm{MgT}$ & $\mathrm{HeSc}$ & MsTr & Ms & Sil & RBU, RLD & + & - \\
\hline $\begin{array}{l}\text { P. chlorantha (Cust.) Rchb. } \\
\text { Poaceae }\end{array}$ & $\mathrm{G}$ & $\mathrm{MgT}$ & $\mathrm{HeSc}$ & $\operatorname{MsTr}$ & Ms & Sil & RBU, RLD & + & - \\
\hline Agropyron lavrencoanum Procud. & $\mathrm{HKr}$ & MsT & $\mathrm{He}$ & $\operatorname{OgTr}$ & MsX & Ps & - & + & - \\
\hline A. pectinatum (Bieb.) Beaux. & $\mathrm{HKr}$ & $\mathrm{MgT}$ & $\mathrm{He}$ & MsTr & $\mathrm{X}$ & St & - & - & + \\
\hline Agrostis gigantea Roth & $\mathrm{HKr}$ & MsT & $\mathrm{ScHe}$ & $\mathrm{MsTr}$ & Ms & SilPr & - & + & - \\
\hline A. stolonifera L. & $\mathrm{HKr}$ & $\mathrm{MgT}$ & $\mathrm{ScHe}$ & OgMsTr & $\mathrm{Hg}$ & PrPal & - & + & + \\
\hline A. vinealis Schreb. & $\mathrm{HKr}$ & MsT & $\mathrm{ScHe}$ & $\mathrm{OgTr}$ & $\mathrm{Ms}$ & StSMnPs & - & + & - \\
\hline Alopecurus aequalis Sobol. & $\mathrm{T}$ & $\mathrm{MgT}$ & $\mathrm{He}$ & $\mathrm{OgTr}$ & HgHel & PrPal & - & + & + \\
\hline A. arundinaceus Poir. & $\mathrm{G}$ & $\mathrm{MgT}$ & $\mathrm{He}$ & AlkMgTr & HgMs & HalPalPr & - & + & + \\
\hline A. geniculatus L. & $\mathrm{T}$ & $\mathrm{MgT}$ & $\mathrm{He}$ & $\mathrm{Og} \operatorname{Tr}$ & HgMs & PalPr & - & + & + \\
\hline A. pratensis $\mathrm{L}$. & $\mathrm{HKr}$ & $\mathrm{MgT}$ & $\mathrm{He}$ & $\operatorname{Mg} \operatorname{Tr}$ & HgMs & $\operatorname{Pr}$ & - & + & + \\
\hline Anisantha sterilis (L.) Nevski & $\mathrm{T}$ & $\mathrm{MgT}$ & $\mathrm{ScHe}$ & MsTr & MsX & PrStRu & Adv & + & + \\
\hline A. tectorum (L.) Nevsli & $\mathrm{T}$ & MgT & $\mathrm{ScHe}$ & OgMgTr & $\mathrm{Ms} \mathrm{X}$ & $\mathrm{PsRu}$ & Adv & + & + \\
\hline Anthoxantum odoratum L. & $\mathrm{HKr}$ & MsT & $\mathrm{HeSc}$ & $\mathrm{OgTr}$ & Ms & SilPr & RBU, RLD & + & + \\
\hline Apera spica-venti (L.) Beauv. & $\mathrm{T}$ & MsT & $\mathrm{ScHe}$ & $\operatorname{OgTr}$ & $\mathrm{XMs}$ & RuPs & Adv & + & + \\
\hline Arrhenatherum elatius (L.) J. et C. Presl & $\mathrm{HKr}$ & $\mathrm{MgT}$ & $\mathrm{ScHe}$ & MsTr & $\mathrm{XMs}$ & SilPr & - & + & + \\
\hline Beckmania eruciformis (L.) Host. & $\mathrm{HKr}$ & MgT & $\mathrm{ScHe}$ & AlkMsTr & HgMs & PalPr & - & + & + \\
\hline Brachypodium sylvaticum (Huds.) P. Beauv. & $\mathrm{HKr}$ & $\operatorname{MgT}$ & $\mathrm{Sc}$ & $\operatorname{MgTr}$ & Ms & Sil & - & + & - \\
\hline Briza media L. & $\mathrm{HKr}$ & $\mathrm{MgT}$ & $\mathrm{HeSc}$ & MsTr & HgMs & SilPr & RLD & + & - \\
\hline Bromopsis inermis (Leys.) Holub & $\mathrm{G}$ & $\operatorname{MgT}$ & $\mathrm{He}$ & OgMgTr & $\mathrm{XMs}$ & RuPrSt & - & + & + \\
\hline B. riparia (Rehm.) Holub & $\mathrm{G}$ & $\mathrm{MgT}$ & $\mathrm{He}$ & $\operatorname{OgTr}$ & $\mathrm{Ms} \mathrm{X}$ & PrSt & - & + & + \\
\hline B. arvensis $\mathrm{L}$. & $\mathrm{T}$ & $\mathrm{MgT}$ & $\mathrm{He}$ & MsTr & $\mathrm{XMs}$ & $\mathrm{Ru}$ & Adv & + & + \\
\hline B. commutatus Schrad & $\mathrm{T}$ & $\mathrm{MgT}$ & $\mathrm{He}$ & MsTr & $\mathrm{XMs}$ & $\mathrm{Ru}$ & Adv & + & + \\
\hline B. hordeaceus L. & $\mathrm{T}$ & $\operatorname{MgT}$ & $\mathrm{ScHe}$ & MsTr & XMs & $\mathrm{Ru}$ & - & + & + \\
\hline B. squarrosus L. & $\mathrm{T}$ & $\operatorname{MgT}$ & $\mathrm{ScHe}$ & OgMgTr & $\mathrm{MsX}$ & RuPsSt & Adv & + & + \\
\hline Calamagrostis canescens (Web.) Roth & $\mathrm{HKr}$ & $\operatorname{MgT}$ & $\mathrm{ScHe}$ & MsTr & $\mathrm{MsHg}$ & SilPrPal & - & + & - \\
\hline C. epigeios (L.)Roth & $\mathrm{G}$ & $\mathrm{MgT}$ & $\mathrm{ScHe}$ & OgMsTr & Ms & PsSilPr & - & + & + \\
\hline Catabrosa aquatica (L.) Beauv. & $\mathrm{HKr}$ & $\operatorname{MgT}$ & $\mathrm{He}$ & MsTr & Hel & PrPal & - & + & + \\
\hline Crypsis aculeata (L.) Ait. & $\mathrm{T}$ & $\operatorname{MgT}$ & $\mathrm{He}$ & AlkMsTr & HgMs & HalPr & RLD & - & + \\
\hline C. schoenoides (L.) Lam. & $\mathrm{T}$ & $\operatorname{MgT}$ & $\mathrm{He}$ & AlkOgTr & Ms & HalPsPr & - & - & + \\
\hline Cynodon dactylon (L.) Pers. & $\mathrm{HKr}$ & $\mathrm{MgT}$ & $\mathrm{He}$ & AlkMsTr & $\mathrm{XMs}$ & $\mathrm{HalPr}$ & Adv & - & + \\
\hline Dactylis glomerata L. & $\mathrm{HKr}$ & $\operatorname{MgT}$ & $\mathrm{ScHe}$ & OgMsTr & Ms & SilPr & - & + & + \\
\hline Digitaria sanguinalis (L.) Scop. & $\mathrm{T}$ & MsT & $\mathrm{He}$ & OgMsTr & Ms & $\mathrm{PsRu}$ & Adv & + & + \\
\hline Echinochloa crusgalli (L.) Beauv. & $\mathrm{T}$ & MsT & $\mathrm{He}$ & OgMgTr & $\mathrm{MsHg}$ & $\mathrm{Ru}$ & Adv & + & + \\
\hline Elymus caninus (L.) L. & $\mathrm{HKr}$ & $\mathrm{MgT}$ & $\mathrm{HeSc}$ & $\operatorname{MgTr}$ & Ms & Sil & - & + & - \\
\hline Elytrigia intermedia (Host) Nevski & $\mathrm{HKr}$ & $\operatorname{MgT}$ & $\mathrm{ScHe}$ & OgMsTr & MsX & StPtPs & - & + & - \\
\hline E. repens (L.) Nevski & $\mathrm{G}$ & MgT & $\mathrm{ScHe}$ & MsTr & Ms & SilStPrRu & - & + & + \\
\hline E. trichophora (Link) Nevski & $\mathrm{G}$ & MgT & $\mathrm{ScHe}$ & MsTr & $\mathrm{XMs}$ & SilSt & - & + & + \\
\hline Eragrostis minor Host & $\mathrm{T}$ & $\operatorname{MgT}$ & $\mathrm{He}$ & OgMsTr & MsX & $\mathrm{PsRu}$ & Adv & + & + \\
\hline Festuca arietina Klokov & $\mathrm{HKr}$ & MsT & $\mathrm{ScHe}$ & $\mathrm{Og} \operatorname{Tr}$ & MsX & SilPs & - & + & - \\
\hline F. beckeri (Hack) Trautv. & $\mathrm{HKr}$ & $\operatorname{MgT}$ & $\mathrm{He}$ & OgTr & $\mathrm{X}$ & StSilPs & - & + & + \\
\hline F. gigantea (L.) Vill. & $\mathrm{HKr}$ & $\operatorname{MgT}$ & $\mathrm{Sc}$ & $\operatorname{MgTr}$ & HgMs & Sil & - & + & - \\
\hline F. pratensis Huds. & $\mathrm{HKr}$ & $\operatorname{MgT}$ & $\mathrm{ScHe}$ & MsTr & HgMs & $\operatorname{Pr}$ & - & + & + \\
\hline F. regeliana Pavl. & $\mathrm{HKr}$ & $\mathrm{MgT}$ & $\mathrm{He}$ & AlkTr & $\mathrm{MsHg}$ & HalPr & - & - & + \\
\hline F. rupicola Heuff. & $\mathrm{HKr}$ & $\operatorname{MgT}$ & $\mathrm{He}$ & $\operatorname{MgTr}$ & $\mathrm{XMs}$ & PrSt & - & + & + \\
\hline F. valesiaca Gaudin. & $\mathrm{HKr}$ & $\operatorname{MgT}$ & $\mathrm{He}$ & $\operatorname{MgTr}$ & $\mathrm{X}$ & St & - & + & + \\
\hline Glyceria arundinacea Kunth & $\mathrm{HKr}$ & $\operatorname{MgT}$ & $\mathrm{He}$ & MsTr & HgHel & PrPal & - & + & + \\
\hline G. fluitans (L.) R.Br. & $\mathrm{HKr}$ & $\mathrm{MgT}$ & $\mathrm{He}$ & MsTr & Hel & PalAq & - & + & + \\
\hline G. maxima (C. Hartm.) Holub. & $\mathrm{HKr}$ & $\operatorname{MgT}$ & $\mathrm{He}$ & MsTr & HgHel & PalAq & - & + & + \\
\hline G. notata Chevall. & $\mathrm{HKr}$ & MsT & $\mathrm{He}$ & $\mathrm{MsTr}$ & $\mathrm{Hg}$ & PrPal & - & + & + \\
\hline Helictotrichon pubescens (Huds.) Pilg. & $\mathrm{HKr}$ & $\operatorname{MgT}$ & $\mathrm{He}$ & MsTr & $\mathrm{XMs}$ & $\mathrm{StPr}$ & RLD & + & - \\
\hline Hierochloe odorata (L.) Beauv. & $\mathrm{G}$ & $\operatorname{MgT}$ & $\mathrm{ScHe}$ & OgMsTr & $\mathrm{XMs}$ & SilStPr & - & + & - \\
\hline H. repens (Host) Beauv. & $\mathrm{G}$ & MsT & $\mathrm{ScHe}$ & OgMsTr & $\mathrm{XMs}$ & PsStPr & - & + & - \\
\hline Koeleria delavignei Gern. ex Domin & $\mathrm{HKr}$ & $\operatorname{MgT}$ & $\mathrm{He}$ & AlkMsTr & $\mathrm{XMs}$ & PrSt & - & - & + \\
\hline K. sabuletorum (Domin) Klokov & $\mathrm{HKr}$ & $\operatorname{MgT}$ & $\mathrm{He}$ & $\mathrm{OgTr}$ & $\mathrm{MsX}$ & PsSt & - & + & + \\
\hline Leersia orizoides (L.) Sw. & $\mathrm{G}$ & MsT & $\mathrm{HeSc}$ & OgMsTr & $\mathrm{HelHg}$ & PrPal & RLD & + & + \\
\hline Lolium perenne $\mathrm{L}$. & $\mathrm{HKr}$ & $\mathrm{MgT}$ & $\mathrm{He}$ & $\operatorname{MgTr}$ & $\mathrm{XMs}$ & $\mathrm{RuPr}$ & - & + & + \\
\hline Melica altissima L. & $\mathrm{HKr}$ & $\mathrm{MgT}$ & $\mathrm{ScHe}$ & MsTr & $\mathrm{XMs}$ & $\mathrm{SMr}$ & - & + & - \\
\hline M. nutans L. & $\mathrm{HKr}$ & $\operatorname{MgT}$ & $\mathrm{Sc}$ & $\mathrm{MsTr}$ & Ms & Sil & RLD & + & - \\
\hline M. picta C.Koch & $\mathrm{HKr}$ & MgT & $\mathrm{ScHe}$ & CaMsTr & $\mathrm{XMs}$ & Sil & - & + & - \\
\hline M. transsilvanica Schur & $\mathrm{HKr}$ & $\operatorname{MgT}$ & $\mathrm{ScHe}$ & CaMsTr & $\mathrm{MsX}$ & SMnSt & - & + & + \\
\hline Milium effusum L. & $\mathrm{HKr}$ & $\operatorname{MgT}$ & $\mathrm{Sc}$ & $\operatorname{MgTr}$ & Ms & Sil & - & + & - \\
\hline Molinia caerulea (L.) Moench & $\mathrm{HKr}$ & MsT & $\mathrm{ScHe}$ & OgTr & $\mathrm{Hg}$ & SilPrPal & RLD & + & - \\
\hline Nardus stricta L. & $\mathrm{HKr}$ & $\mathrm{MgT}$ & $\mathrm{He}$ & OgTr & $\mathrm{Hg}$ & SilPalPr & RLD & + & - \\
\hline Phalaroides arundinacea (L.) Rauschert. & $\mathrm{HKr}$ & $\operatorname{MgT}$ & $\mathrm{ScHe}$ & $\operatorname{MgTr}$ & $\mathrm{MsHg}$ & PrPal & - & + & + \\
\hline Phleum phleoides (L.) Karst. & $\mathrm{HKr}$ & $\operatorname{MgT}$ & $\mathrm{He}$ & MsTr & $\mathrm{XMs}$ & PrSt & - & + & + \\
\hline Phragmites australis (Cav.) Trin.ex Steud. & $\mathrm{HKr}$ & EuT & $\mathrm{ScHe}$ & MsTr & Hel & PalAq & - & + & + \\
\hline Poa angustifolia $\mathrm{L}$. & $\mathrm{HKr}$ & $\mathrm{MgT}$ & $\mathrm{ScHe}$ & $\operatorname{MsMgTr}$ & MsX & SilPrSt & - & + & + \\
\hline P. аппиа $\mathrm{L}$. & $\mathrm{T}$ & EuT & $\mathrm{HeSc}$ & MsTr & Ms & RuSilPr & - & + & + \\
\hline P. bulbosa L. & $\mathrm{HKr}$ & EuT & $\mathrm{He}$ & OgMsTr & MsX & RuSilSt & - & + & + \\
\hline P. compressa $\mathrm{L}$. & $\mathrm{HKr}$ & $\operatorname{MgT}$ & $\mathrm{ScHe}$ & OgMsTr & MsX & RuSt & - & - & + \\
\hline P. nemoralis L. & $\mathrm{HKr}$ & $\mathrm{MgT}$ & $\mathrm{ScHe}$ & MsTr & $\mathrm{XMs}$ & Sil & - & + & - \\
\hline P. palustris L. & $\mathrm{HKr}$ & MsT & $\mathrm{He}$ & MsTr & $\mathrm{MsHg}$ & PalPr & - & + & + \\
\hline
\end{tabular}




\begin{tabular}{|c|c|c|c|c|c|c|c|c|c|}
\hline 1 & 2 & 3 & 4 & 5 & 6 & 7 & 8 & 9 & 10 \\
\hline P. pratensis L. & G & MsT & $\mathrm{He}$ & MsTr & Ms & $\operatorname{Pr}$ & - & + & + \\
\hline P. remota Forcelles & $\mathrm{HKr}$ & MsT & $\mathrm{ScHe}$ & MsTr & $\mathrm{HgMs}$ & Sil & RLD & + & - \\
\hline P. sylvicola Guss. & $\mathrm{HKr}$ & $\operatorname{MgT}$ & $\mathrm{HeSc}$ & $\operatorname{MgTr}$ & $\mathrm{HgMs}$ & SilPalPr & - & + & - \\
\hline P. trivialis $\mathrm{L}$. & $\mathrm{HKr}$ & MsT & $\mathrm{He}$ & MsTr & HgMs & SilPalPr & - & + & - \\
\hline Puccinella bilykiana Klokov & $\mathrm{HKr}$ & MsT & $\mathrm{He}$ & AlkTr & Ms & HalPr & - & - & + \\
\hline P. distans (Jacq.) Parl. & $\mathrm{HKr}$ & MsT & $\mathrm{He}$ & AlkMsTr & $\mathrm{XMs}$ & RuHalPr & - & - & + \\
\hline Scolochloa festucacea (Willd.) Link. & $\mathrm{HKr}$ & MsT & $\mathrm{He}$ & MsTr & $\mathrm{Hg}$ & Pal & RLD & + & + \\
\hline Setaria glauca (L.) Beauv. & $\mathrm{T}$ & $\mathrm{MgT}$ & $\mathrm{He}$ & MsTr & $\mathrm{XMs}$ & PsRu & Adv & + & + \\
\hline S. verticillata (L.) H. B. & $\mathrm{T}$ & MgT & $\mathrm{ScHe}$ & $\operatorname{MgTr}$ & Ms & $\mathrm{Ru}$ & Adv & + & + \\
\hline \multicolumn{10}{|l|}{ Potamogetonaceae } \\
\hline Potamogeton berchtoldii Fieb. & $\mathrm{HKr}$ & MsT & HeSc & MsTr & Hy & $\mathrm{Aq}$ & - & + & + \\
\hline P. compressus $\mathrm{L}$. & $\mathrm{HKr}$ & MsT & $\mathrm{HeSc}$ & MsTr & Hy & $\mathrm{Aq}$ & - & + & - \\
\hline P. crispus $\mathrm{L}$. & $\mathrm{HKr}$ & EuT & $\mathrm{HeSc}$ & MsTr & Hy & $\mathrm{Aq}$ & - & + & + \\
\hline P. gramineus L. & $\mathrm{HKr}$ & $\mathrm{MgT}$ & $\mathrm{ScHe}$ & MsTr & PIHy & $\mathrm{Aq}$ & - & + & + \\
\hline P. lucens L. & $\mathrm{HKr}$ & $\mathrm{MgT}$ & $\mathrm{HeSc}$ & MsTr & Hy & $\mathrm{Aq}$ & - & + & - \\
\hline P. natans L. & $\mathrm{HKr}$ & MsT & $\mathrm{ScHe}$ & MsTr & $\mathrm{Pl}$ & $\mathrm{Aq}$ & RLD & + & - \\
\hline P. nodosus Poir. & $\mathrm{HKr}$ & $\mathrm{MgT}$ & $\mathrm{ScHe}$ & MsTr & Hy & $\mathrm{Aq}$ & RLD & + & - \\
\hline P. pectinatus $\mathrm{L}$. & $\mathrm{HKr}$ & EuT & $\mathrm{HeSc}$ & $\mathrm{MsTr}$ & Hy & $\mathrm{Aq}$ & - & + & + \\
\hline P. perfoliatus L. & $\mathrm{HKr}$ & $\operatorname{MgT}$ & $\mathrm{HeSc}$ & MsTr & Hy & $\mathrm{Aq}$ & - & + & + \\
\hline $\begin{array}{l}\text { P. sarmaticus Maemets } \\
\text { Sparganiaceae }\end{array}$ & $\mathrm{HKr}$ & MsT & $\mathrm{HeSc}$ & $\mathrm{MsTr}$ & Hy & $\mathrm{Aq}$ & RLD & + & - \\
\hline Sparganium erectum $\mathrm{L}$. & $\mathrm{HKr}$ & $\operatorname{MgT}$ & $\mathrm{He}$ & MsTr & Hel & PalAq & - & + & + \\
\hline S. minimum Wallr. & $\mathrm{HKr}$ & MsT & $\mathrm{HeSc}$ & $\mathrm{MsTr}$ & Hel & PalAq & RLD & + & + \\
\hline Typha angustifolia $\mathrm{L}$. & $\mathrm{HKr}$ & EuT & $\mathrm{He}$ & MsTr & Hel & PalAq & - & + & + \\
\hline T. latifolia $\mathrm{L}$. & $\mathrm{HKr}$ & MsT & $\mathrm{He}$ & $\operatorname{MgTr}$ & Hel & PalAq & - & + & + \\
\hline $\begin{array}{l}\text { T. laxmannii Lepech. } \\
\text { Zannicheliaceae }\end{array}$ & $\mathrm{HKr}$ & MsT & $\mathrm{He}$ & AlkMsTr & Hel & PalAq & - & + & + \\
\hline $\begin{array}{l}\text { Zannichelia palustris L. } \\
\text { Клас Magnoliopsida } \\
\text { Aceraceae }\end{array}$ & $\mathrm{HKr}$ & $\operatorname{MgT}$ & $\mathrm{HeSc}$ & AlkMsTr & Hy & $\mathrm{Aq}$ & RLD & - & + \\
\hline Acer campestre L. & $\mathrm{Ph}$ & MgT & $\mathrm{ScHe}$ & MgMsTr & XMs & SMnSil & - & + & - \\
\hline A. negundo L. & $\mathrm{Ph}$ & MsT & $\mathrm{He}$ & MsTr & Ms & SilCuRu & Adv & + & + \\
\hline A. platanoides $\mathrm{L}$. & $\mathrm{Ph}$ & MsT & $\mathrm{HeSc}$ & MgMsTr & Ms & Sil & - & + & - \\
\hline A. tataricum $\mathrm{L}$. & $\mathrm{Ph}$ & MsT & $\mathrm{ScHe}$ & AlkMsTr & Ms & SilSMn & - & + & + \\
\hline $\begin{array}{l}\text { Adoxaceae } \\
\text { Adoxa moschatellina } \mathrm{L} \text {. } \\
\text { Amaranthaceae }\end{array}$ & $\mathrm{HKr}$ & MsT & $\mathrm{HeSc}$ & $\operatorname{Ms} T r$ & Ms & Sil & RLD & + & - \\
\hline Amaranthus albus L. & $\mathrm{T}$ & MsT & $\mathrm{He}$ & MsTr & MsX & $\mathrm{Ru}$ & Adv & + & + \\
\hline A. blitoides S.Wats. & $\mathrm{T}$ & $\operatorname{MgT}$ & $\mathrm{He}$ & MsTr & MsX & $\mathrm{Ru}$ & Adv & + & + \\
\hline A. caudatus $\mathrm{L}$. & $\mathrm{T}$ & MgT & $\mathrm{He}$ & $\operatorname{MgTr}$ & $\mathrm{XMs}$ & $\mathrm{CuRu}$ & Adv & + & + \\
\hline A. retroflexus L. & $\mathrm{T}$ & MsT & $\mathrm{He}$ & MsTr & MsX & $\mathrm{Ru}$ & Adv & + & + \\
\hline \multicolumn{10}{|l|}{ Apiaceae } \\
\hline Aegopodium podagraria L. & G & MsT & $\mathrm{HeSc}$ & $\operatorname{MgTr}$ & Ms & Sil & - & + & - \\
\hline Aethusa cynapium L. & $\mathrm{THKr}$ & $\mathrm{MgT}$ & $\mathrm{ScHe}$ & MsTr & $\mathrm{XMs}$ & SilRu & Adv & + & - \\
\hline Antriscus cerefolium (L.) Hoffm. & $\mathrm{T}$ & MgT & $\mathrm{HeSc}$ & $\mathrm{MsTr}$ & $\mathrm{XMs}$ & SilRu & - & + & - \\
\hline Antriscus sylvestris (L.) Hoffm. & $\mathrm{HKr}$ & MsT & $\mathrm{HeSc}$ & MsTr & Ms & RuSil & - & + & + \\
\hline Chaerophyllum bulbosum $\mathrm{L}$. & $\mathrm{HKr}$ & MsT & $\mathrm{ScHe}$ & $\mathrm{Og} \operatorname{Tr}$ & Ms & PrSil & - & + & - \\
\hline Chaerophyllum temulum L. & $\mathrm{THKr}$ & MsT & $\mathrm{HeSc}$ & MsTr & Ms & RuSil & - & + & - \\
\hline Cicuta virosa $\mathrm{L}$. & Hel & MsT & $\mathrm{HeSc}$ & $\mathrm{MsTr}$ & $\mathrm{Hg}$ & Pal & - & + & - \\
\hline Conium maculatum $\mathrm{L}$. & $\mathrm{HKr}$ & MgT & $\mathrm{He}$ & MsTr & $\mathrm{Ms}$ & $\mathrm{Ru}$ & - & + & - \\
\hline Daucus carota L. & $\mathrm{HKrT}$ & MgT & $\mathrm{ScHe}$ & $\mathrm{MsTr}$ & $\mathrm{XMs}$ & $\mathrm{Ru}$ & - & + & + \\
\hline Eryngium campestre L. & G & MgT & $\mathrm{He}$ & MsTr & $\mathrm{X}$ & RuSt & - & + & + \\
\hline E. planum $\mathrm{L}$. & $\mathrm{HKr}$ & MgT & $\mathrm{ScHe}$ & Og-MsTr & $\mathrm{XMs}$ & StPr & - & + & + \\
\hline Heracleum sibiricum L. & $\mathrm{HKr}$ & MsT & $\mathrm{ScHe}$ & MsTr & Ms & SilPr & - & + & + \\
\hline Laser trilobum (L.) Borkh. & $\mathrm{HKr}$ & $\operatorname{MgT}$ & $\mathrm{ScHe}$ & $\operatorname{MsTr}$ & Ms & Sil & RLD & + & - \\
\hline Oenanthe aquatica (L.) Poir. & $\mathrm{HKr}$ & MsT & $\mathrm{ScHe}$ & MsTr & $\mathrm{Hg}$ & Pal & - & + & + \\
\hline Pastinaca sylvestris Mill. & $\mathrm{HKr}$ & MsT & $\mathrm{ScHe}$ & $\operatorname{MsTr}$ & Ms & SilPr & - & + & + \\
\hline Peucedanum latifolium (Bieb.) DC. & $\mathrm{HKr}$ & $\mathrm{MgT}$ & $\mathrm{He}$ & AlkTr & Ms & $\mathrm{PrH}$ & - & - & + \\
\hline P. lubimencoanum Kotov & $\mathrm{HKr}$ & $\mathrm{MgT}$ & $\mathrm{HeSc}$ & $\operatorname{Ms} T r$ & $\mathrm{XMs}$ & SMn & - & + & - \\
\hline P. palustre (L.) Moench & $\mathrm{HKr}$ & MsT & $\mathrm{He}$ & $\operatorname{MgTr}$ & $\mathrm{MsHg}$ & PrPal & RLD & + & + \\
\hline Siella erecta (Huds.) M. Pimen. & $\mathrm{HKr}$ & MsT & $\mathrm{He}$ & OgTr & $\mathrm{Hg}$ & Pal & RLD & + & + \\
\hline Sium latifolium $\mathrm{L}$. & $\mathrm{HKr}$ & MsT & $\mathrm{ScHe}$ & MsTr & $\mathrm{Hg}$ & $\mathrm{Pal}$ & - & + & + \\
\hline S. sisaroideum DC. & $\mathrm{HKr}$ & $\mathrm{MgT}$ & $\mathrm{He}$ & MsTr & $\mathrm{Hg}$ & Pal & - & + & + \\
\hline $\begin{array}{l}\text { Torilis japonijca (Houtt.) DC. } \\
\text { Aristolochiaceae }\end{array}$ & \multicolumn{8}{|c|}{ Aristolochiaceae } & + \\
\hline Aristolochia clematitis L. & G & $\mathrm{MgT}$ & $\mathrm{HeSc}$ & MsTr & $\mathrm{HgMs}$ & RuPrSil & - & + & + \\
\hline Asarum europaeum L. & $\mathrm{G}$ & MsT & $\mathrm{Sc}$ & $\operatorname{MgTr}$ & Ms & Sil & RLD & + & - \\
\hline \multicolumn{10}{|l|}{ Asclepiadaceae } \\
\hline Asclepias syriaca L. & $\mathrm{G}$ & MgT & $\mathrm{He}$ & MsTr & $\mathrm{XMs}$ & $\mathrm{Ru}$ & Adv & - & + \\
\hline $\begin{array}{c}\text { Vincetoxicum hirundinaria Medik. } \\
\text { Asteraceae }\end{array}$ & $\mathrm{HKr}$ & $\mathrm{MgT}$ & $\mathrm{ScHe}$ & $\mathrm{MsTr}$ & MsX & StSMnSil & - & + & + \\
\hline Achillea setacea Waldst.et Kit. & $\mathrm{HKr}$ & MsT & $\mathrm{ScHe}$ & MsTr & $\mathrm{X}$ & StSilSMn & - & + & + \\
\hline A. submillefolium Klok. et Krytzka & $\mathrm{HKr}$ & MsT & $\mathrm{ScHe}$ & MsTr & $\mathrm{XMs}$ & SilSMnPrSt & - & + & + \\
\hline Ambrosia artemisifolia L. & $\mathrm{T}$ & MsT & $\mathrm{ScHe}$ & OgMgTr & $\mathrm{X}$ & $\mathrm{Ru}$ & Adv & + & + \\
\hline Anthemis cotula L. & $\mathrm{T}$ & $\mathrm{MgT}$ & $\mathrm{He}$ & MsTr & $\mathrm{XMs}$ & $\mathrm{Ru}$ & Adv & + & + \\
\hline
\end{tabular}




\begin{tabular}{|c|c|c|c|c|c|c|c|c|c|}
\hline 1 & 2 & 3 & 4 & 5 & 6 & 7 & 8 & 9 & 10 \\
\hline Arctium lappa L. & $\mathrm{HKr}$ & MsT & $\mathrm{ScHe}$ & $\operatorname{MgTr}$ & Ms & SilRu & - & + & + \\
\hline A. nemorosum Lej. & $\mathrm{HKr}$ & MsT & $\mathrm{HeSc}$ & $\operatorname{MgTr}$ & Ms & RuSil & - & + & - \\
\hline A. tomentosum Mill. & $\mathrm{HKr}$ & MsT & $\mathrm{He}$ & $\operatorname{MgTr}$ & Ms & $\mathrm{Ru}$ & - & + & + \\
\hline Artemisia abrotanum L. & $\mathrm{Ch}$ & MsT & $\mathrm{He}$ & OgMsTr & $\mathrm{HgMs}$ & PalPr & - & + & + \\
\hline A. absinthium L. & $\mathrm{HKr}$ & MsT & $\mathrm{He}$ & $\mathrm{MsTr}$ & $\mathrm{XMs}$ & $\mathrm{Ru}$ & Adv & + & + \\
\hline A. austriaca Jacq. & $\mathrm{Ch}$ & MsT & $\mathrm{He}$ & $\mathrm{MsTr}$ & X & RuSt & - & + & + \\
\hline A. campestris $\mathrm{L}$. & $\mathrm{HKr}$ & MsT & $\mathrm{He}$ & OgMsTr & $\mathrm{X}$ & SilPsSt & - & + & + \\
\hline A. pontica $\mathrm{L}$. & $\mathrm{HKr}$ & MsT & $\mathrm{ScHe}$ & MsTr & $\mathrm{X}$ & SilSMnSt & RLD & + & + \\
\hline A. vulgaris $\mathrm{L}$. & $\mathrm{HKr}$ & MsT & $\mathrm{ScHe}$ & $\operatorname{MgTr}$ & Ms & PrRu & - & + & + \\
\hline Bidens tripartita $\mathrm{L}$. & $\mathrm{T}$ & EuT & $\mathrm{ScHe}$ & $\mathrm{MsTr}$ & $\mathrm{HgMs}$ & $\mathrm{PalPr}$ & - & + & + \\
\hline Carduus acanthoides L. & $\mathrm{THKr}$ & MsT & $\mathrm{He}$ & $\mathrm{Ms} T r$ & MsX & $\mathrm{PrStRu}$ & - & + & + \\
\hline C. crispus $\mathrm{L}$. & $\mathrm{HKr}$ & MsT & $\mathrm{ScHe}$ & MsTr & Ms & SilRu & - & + & - \\
\hline C. uncinatus M. Bieb. & $\mathrm{HKr}$ & MsT & $\mathrm{He}$ & $\operatorname{MgTr}$ & MsX & RuSt & - & - & + \\
\hline Centaurea diffusa Lam. & $\mathrm{HKr}$ & $\mathrm{MgT}$ & $\mathrm{He}$ & MsTr & X & $\mathrm{StRu}$ & Adv & - & + \\
\hline C. jacea $\mathrm{L}$. & $\mathrm{HKr}$ & MsT & $\mathrm{ScHe}$ & $\operatorname{MgTr}$ & Ms & $\operatorname{Pr}$ & - & + & + \\
\hline C. scabiosa L. & $\mathrm{HKr}$ & MsT & $\mathrm{ScHe}$ & $\operatorname{MgTr}$ & MsX & $\mathrm{StRu}$ & - & + & + \\
\hline C. substituta Czerep. & $\mathrm{HKr}$ & MsT & $\mathrm{ScHe}$ & $\mathrm{Ms} \operatorname{Tr}$ & Ms & SilPr & RLD & + & + \\
\hline C. trichocephala Bieb. & $\mathrm{HKr}$ & $\mathrm{MgT}$ & $\mathrm{ScHe}$ & MsTr & $\mathrm{XMs}$ & PrSt & - & + & + \\
\hline Chartolepis intermedia Boiss. & $\mathrm{G}$ & $\mathrm{MgT}$ & $\mathrm{He}$ & AlkTr & Ms & PrHal & - & - & + \\
\hline Cichorium inthybus L. & $\mathrm{HKr}$ & MsT & $\mathrm{He}$ & MsTr & MsX & RuStPr & Adv & + & + \\
\hline Cirsium alatum (S.B.Gmel.)Bobr. & $\mathrm{HKr}$ & MsT & $\mathrm{He}$ & AlkTr & Ms & PrHal & RLD & + & + \\
\hline C. polonicum (Petrak) Iljin & $\mathrm{HKr}$ & MsT & $\mathrm{He}$ & MsTr & MsX & $\mathrm{StRu}$ & - & - & + \\
\hline C. rivulare (Jacq.) All. & $\mathrm{HKr}$ & MsT & $\mathrm{He}$ & $\mathrm{Ms} \operatorname{Tr}$ & $\mathrm{MsHg}$ & PalPr & RLD & + & + \\
\hline C. setosum (Willd.) Bess. & $\mathrm{G}$ & $\mathrm{MgT}$ & $\mathrm{He}$ & MsTr & MsX & $\mathrm{Ru}$ & - & + & + \\
\hline C.ucrainicum Bess. & $\mathrm{HKr}$ & MsT & $\mathrm{He}$ & OgMsTr & $\mathrm{X}$ & RuSt & - & - & + \\
\hline C. vulgare (Savit) Ten. & $\mathrm{HKr}$ & $\mathrm{MgT}$ & $\mathrm{ScHe}$ & $\mathrm{MgTr}$ & $\mathrm{XMs}$ & $\mathrm{Ru}$ & - & + & + \\
\hline Coniza canadensis (L.) Cronq. & $\mathrm{THKr}$ & EuT & $\mathrm{ScHe}$ & $\mathrm{OgMgTr}$ & MsX & $\mathrm{Ru}$ & Adv & + & + \\
\hline Crepis rhoedifolia M.Bieb. & $\mathrm{T}$ & $\mathrm{MgT}$ & $\mathrm{He}$ & $\mathrm{MsTr}$ & MsX & $\mathrm{StRu}$ & - & - & + \\
\hline C. setosa Hal. & $\mathrm{T}$ & MsT & $\mathrm{He}$ & $\mathrm{MsTr}$ & MsX & $\mathrm{PrRu}$ & - & + & + \\
\hline C. tectorum $\mathrm{L}$. & $\mathrm{THKr}$ & MsT & $\mathrm{He}$ & OgMsTr & MsX & PsStRu & - & + & + \\
\hline Echinops ruthenicus M. Bieb. & $\mathrm{HKr}$ & MsT & $\mathrm{ScHe}$ & $\operatorname{MgTr}$ & $\mathrm{X}$ & PtSt & - & - & + \\
\hline E. sphaerocephalus L. & $\mathrm{HKr}$ & MsT & $\mathrm{ScHe}$ & MsTr & $\mathrm{X}$ & SMnSt & - & + & + \\
\hline Erigeron acris L. & $\mathrm{HKr}$ & MsT & $\mathrm{ScHe}$ & $\mathrm{MsTr}$ & MsX & RuPrSt & - & - & + \\
\hline Eupatorium cannabinum $\mathrm{L}$. & $\mathrm{HKr}$ & EuT & $\mathrm{ScHe}$ & $\operatorname{MgTr}$ & $\mathrm{HgMs}$ & SilPr & - & + & - \\
\hline Filago arvensis $\mathrm{L}$. & $\mathrm{T}$ & $\mathrm{MgT}$ & $\mathrm{ScHe}$ & OgMsTr & MsX & SilStRu & - & - & + \\
\hline Galinsoga parviflora Cav. & $\mathrm{T}$ & $\mathrm{MgT}$ & $\mathrm{ScHe}$ & MsTr & Ms & $\mathrm{Ru}$ & Adv & + & + \\
\hline Gnaphalium uliginosum L. & $\mathrm{T}$ & MsT & $\mathrm{He}$ & MsTr & $\mathrm{MsHg}$ & PrPal & RLD & + & - \\
\hline Hieracium umbellatum L. & $\mathrm{HKr}$ & MsT & $\mathrm{ScHe}$ & $\mathrm{Og} \operatorname{Tr}$ & MsX & StPs & RLD & + & + \\
\hline Hypochaeris maculata L. & $\mathrm{HKr}$ & MsT & $\mathrm{ScHe}$ & $\mathrm{MsTr}$ & Ms & SilPr & - & + & - \\
\hline Inula britanica $\mathrm{L}$. & $\mathrm{HKr}$ & $\mathrm{MgT}$ & $\mathrm{He}$ & MsTr & Ms & $\mathrm{RuPr}$ & - & + & + \\
\hline I. germanica L. & $\mathrm{G}$ & $\mathrm{MgT}$ & $\mathrm{He}$ & $\operatorname{MgTr}$ & XMs & StPr & - & - & + \\
\hline I. helenium L. & $\mathrm{HKr}$ & $\mathrm{MgT}$ & $\mathrm{He}$ & $\operatorname{MgTr}$ & $\mathrm{HgMs}$ & Pr & RLD & - & + \\
\hline I. hirta $\mathrm{L}$ & $\mathrm{HKr}$ & MsT & $\mathrm{ScHe}$ & $\operatorname{MgTr}$ & MsX & PrSilSt & RLD & + & + \\
\hline I. salicina $\mathrm{L}$. & $\mathrm{G}$ & $\mathrm{MgT}$ & $\mathrm{ScHe}$ & $\mathrm{Og} \operatorname{Tr}$ & XMs & PrSMn & - & + & + \\
\hline Iva xanthiifolia Nutt. & $\mathrm{T}$ & MsT & $\mathrm{ScHe}$ & OgMgTr & $\mathrm{XMs}$ & $\mathrm{Ru}$ & Adv & + & + \\
\hline Lactuca chaixii Vill. & $\mathrm{T}$ & MsT & $\mathrm{HeSc}$ & $\operatorname{MgTr}$ & Ms & Sil & - & + & - \\
\hline L. serriola Tomer & $\mathrm{THKr}$ & $\mathrm{MgT}$ & $\mathrm{He}$ & MsTr & $\mathrm{XMs}$ & $\mathrm{Ru}$ & Adv & + & + \\
\hline L. quercina $\mathrm{L}$. & $\mathrm{HKr}$ & MsT & $\mathrm{HeSc}$ & $\mathrm{MsTr}$ & Ms & Sil & RLD & + & - \\
\hline L. tatarica (L.) C. A. Mey. & G & $\mathrm{MgT}$ & $\mathrm{He}$ & MsTr & $\mathrm{XMs}$ & $\mathrm{HalRu}$ & - & + & + \\
\hline Lapsana communis L. & $\mathrm{T}$ & $\mathrm{MgT}$ & $\mathrm{HeSc}$ & $\operatorname{MgTr}$ & $\mathrm{XMs}$ & RuSil & - & + & - \\
\hline Leontodon autumnalis $\mathrm{L}$. & $\mathrm{HKr}$ & MsT & $\mathrm{ScHe}$ & MsTr & Ms & $\mathrm{RuPr}$ & - & - & + \\
\hline Lepidotheca suaveolens (Pursh) Nutt. & $\mathrm{T}$ & EuT & $\mathrm{He}$ & $\operatorname{MsTr}$ & MsX & $\mathrm{Ru}$ & Adv & + & + \\
\hline Matricaria recutita $\mathrm{L}$. & $\mathrm{T}$ & MsT & $\mathrm{He}$ & $\operatorname{MsTr}$ & MsX & $\mathrm{Ru}$ & Adv & + & + \\
\hline Onopordum acanthium L. & $\mathrm{HKr}$ & MsT & $\mathrm{He}$ & $\operatorname{MsTr}$ & MsX & $\mathrm{Ru}$ & Adv & - & + \\
\hline Petasites spurius (Retz.) Rchb. & $\mathrm{G}$ & MsT & $\mathrm{ScHe}$ & $\mathrm{Og} \operatorname{Tr}$ & $\mathrm{MsHg}$ & Ps & Adv & + & + \\
\hline Phalacrochena inuloides (Fisch.ex Shmalh.) Iljin & G & MsT & $\mathrm{He}$ & AlkMgTr & $\mathrm{XMs}$ & RuHalPr & RLD & - & + \\
\hline Phalacroloma anmuum (L.) Dumort. & $\mathrm{THKr}$ & MsT & $\mathrm{ScHe}$ & MsTr & MsX & $\mathrm{Ru}$ & Adv & + & + \\
\hline Picris hieracioides L. & $\mathrm{HKr}$ & EuT & $\mathrm{He}$ & $\operatorname{MsTr}$ & MsX & RuSt & - & + & + \\
\hline Pilosella caespitosa (Dumort.) P.D.Seli et West & $\mathrm{HKr}$ & MsT & $\mathrm{He}$ & $\operatorname{MgTr}$ & Ms & $\mathrm{SMnPr}$ & - & + & + \\
\hline Ptarmica salicifolia (Besser) Serg. & $\mathrm{HKr}$ & MsT & $\mathrm{He}$ & OgMsTr & $\mathrm{MsHg}$ & PalPr & - & + & + \\
\hline Pyrethrum corymbosum (L.) Scop. & $\mathrm{HKr}$ & MsT & $\mathrm{ScHe}$ & $\operatorname{MgTr}$ & $\mathrm{XMs}$ & PrSil & RLD & + & - \\
\hline Rhaponticum serratuloides (Georgi) Bobr. & $\mathrm{HKr}$ & $\mathrm{MgT}$ & $\mathrm{He}$ & AlkTr & $\mathrm{MsHg}$ & HalPr & RLD & - & + \\
\hline Scorzonera laciniata L. & $\mathrm{HKr}$ & $\mathrm{MgT}$ & $\mathrm{He}$ & AlkTr & $\mathrm{XMs}$ & HalStPr & - & + & + \\
\hline S. parviflora Jacq. & $\mathrm{HKr}$ & MsT & $\mathrm{He}$ & AlkTr & Ms & HalPr & - & - & + \\
\hline Senecio erucifolius L. & $\mathrm{HKr}$ & MsT & $\mathrm{He}$ & AlkMsTr & $\mathrm{XMs}$ & StHalPr & - & - & + \\
\hline S. grandidentatus Ledeb. & $\mathrm{HKr}$ & $\mathrm{MgT}$ & $\mathrm{ScHe}$ & AlkOgMsTr & $\mathrm{XMs}$ & StPsHalPr & - & - & + \\
\hline Seneciojacobaea L. & $\mathrm{HKr}$ & MsT & $\mathrm{He}$ & MsTr & MsX & RuSMnPrSt & - & + & + \\
\hline S. paucifolius S.G. Gmel. & $\mathrm{HKr}$ & $\mathrm{MgT}$ & $\mathrm{He}$ & AlkTr & $\mathrm{XMs}$ & HalPr & RLD & - & + \\
\hline Serratula coronata L. & $\mathrm{HKr}$ & $\mathrm{MgT}$ & $\mathrm{ScHe}$ & MsTr & Ms & $\mathrm{SMnPr}$ & - & + & + \\
\hline S. lycopifolia (Vill.) A. Kerner & $\mathrm{HKr}$ & $\mathrm{MgT}$ & $\mathrm{ScHe}$ & $\operatorname{MgTr}$ & MsX & StPrSMn & - & + & + \\
\hline Sonchus arvensis L. & $\mathrm{G}$ & EuT & $\mathrm{He}$ & $\operatorname{MgTr}$ & Ms & PrRu & Adv & + & + \\
\hline S. palustris L. & $\mathrm{HKr}$ & MsT & $\mathrm{He}$ & $\mathrm{MsTr}$ & $\mathrm{MsHg}$ & Pal & - & + & - \\
\hline Tanacetum vulgare $\mathrm{L}$. & $\mathrm{HKr}$ & MsT & $\mathrm{He}$ & OgMgTr & MsX & StRuPr & - & + & + \\
\hline Taraxacum officinale Wigg. aggr. & $\mathrm{HKr}$ & EuT & $\mathrm{ScHe}$ & MsTr & Ms & RuPr & - & + & + \\
\hline Tragopogon major Jacq. & $\mathrm{HKr}$ & MsT & $\mathrm{He}$ & MsTr & MsX & SMnRuSt & - & + & + \\
\hline Tragopogon orientalis L. & $\mathrm{HKr}$ & $\mathrm{MgT}$ & $\mathrm{He}$ & $\operatorname{MgTr}$ & $\mathrm{X}$ & St & RLD & + & + \\
\hline Tripleurospermum inodorum (L.) Sch. & $\mathrm{THKr}$ & $\mathrm{MgT}$ & $\mathrm{He}$ & $\operatorname{MgTr}$ & MsX & $\mathrm{Ru}$ & Adv & + & + \\
\hline
\end{tabular}




\begin{tabular}{|c|c|c|c|c|c|c|c|c|c|}
\hline 1 & 2 & 3 & 4 & 5 & 6 & 7 & 8 & 9 & 10 \\
\hline Tussilago farfara $\mathrm{L}$. & G & MsT & $\mathrm{He}$ & MsTr & $\mathrm{MsHg}$ & $\mathrm{RuPr}$ & - & + & + \\
\hline Xantium californicum Greene & $\mathrm{T}$ & MsT & $\mathrm{He}$ & $\operatorname{MsTr}$ & Ms & $\mathrm{Ru}$ & $\mathrm{Adv}$ & + & + \\
\hline$X$. spinosum $\mathrm{L}$. & $\mathrm{T}$ & MsT & $\mathrm{He}$ & MsTr & Ms & $\mathrm{Ru}$ & $\mathrm{Adv}$ & - & + \\
\hline X. strumarium $\mathrm{L}$ & $\mathrm{T}$ & MsT & $\mathrm{He}$ & $\mathrm{MsTr}$ & Ms & $\mathrm{Ru}$ & Adv & + & + \\
\hline $\begin{array}{c}\text { Balsaminaceae } \\
\text { Impatiens noli-tangere } \mathrm{L} \text {. } \\
\text { Betulaceae }\end{array}$ & $\mathrm{T}$ & MsT & $\mathrm{HeSc}$ & $\operatorname{MsTr}$ & $\mathrm{HgMs}$ & PalSil & RLD & + & - \\
\hline $\begin{array}{l}\text { Alnus glutinosa (L.) Gaerthn. } \\
\text { Boraginaceae }\end{array}$ & \multicolumn{8}{|c|}{ Boraginaceae } & - \\
\hline Aegonychon purpureocaeruleum (L.) Holub & $\mathrm{HKr}$ & $\mathrm{MgT}$ & $\mathrm{HeSc}$ & MsMgTr & Ms & Sil & RLD & + & - \\
\hline Asperugo procumbens L. & $\mathrm{T}$ & $\mathrm{MgT}$ & $\mathrm{ScHe}$ & MsTr & $\mathrm{XMs}$ & $\mathrm{Ru}$ & - & + & + \\
\hline Buglossoides arvensis (L.) I.M. Johnst. & $\mathrm{THKr}$ & MgT & $\mathrm{ScHe}$ & $\operatorname{Ms} T r$ & MsX & $\mathrm{Ru}$ & Adv & + & + \\
\hline Cynoglossum officinale $\mathrm{L}$. & G & $\mathrm{MgT}$ & $\mathrm{He}$ & $\mathrm{Og} \operatorname{Tr}$ & $\mathrm{XMs}$ & $\mathrm{Ru}$ & $\mathrm{Adv}$ & - & + \\
\hline Echium vulgare L. & $\mathrm{HKr}$ & MgT & $\mathrm{He}$ & MsTr & $\mathrm{X}$ & PsStRu & - & - & + \\
\hline Lithospermum officinale $\mathrm{L}$. & $\mathrm{T}$ & MsT & $\mathrm{He}$ & $\mathrm{MsTr}$ & $\mathrm{X}$ & RuStPt & - & - & + \\
\hline Lycopsis arvensis L. & $\mathrm{T}$ & MsT & $\mathrm{He}$ & MsTr & X & $\mathrm{RuPt}$ & - & - & + \\
\hline Myosotis laxa Lehm. & $\mathrm{THKr}$ & $\mathrm{MgT}$ & $\mathrm{ScHe}$ & $\mathrm{MsTr}$ & $\mathrm{MsHg}$ & PrPal & - & + & + \\
\hline M. micrantha Pall.ex Lehm. & $\mathrm{HKr}$ & $\mathrm{MgT}$ & $\mathrm{He}$ & $\operatorname{MsTr}$ & MsX & $\underset{\mathrm{t}}{\mathrm{SMnRuPsS}}$ & - & + & + \\
\hline M. nemorosa Besser & $\mathrm{HKr}$ & MsT & $\mathrm{ScHe}$ & MsTr & $\mathrm{MsHg}$ & PalSil & - & + & - \\
\hline M. scorpioides L. & $\mathrm{HKr}$ & MT & $\mathrm{ScHe}$ & MsTr & $\mathrm{Hg}$ & PrPal & - & + & + \\
\hline M. sparsiflora J.C. Mikan ex Pohl & $\mathrm{THKr}$ & $\mathrm{MgT}$ & $\mathrm{ScHe}$ & $\mathrm{MsTr}$ & MsX & RuSil & - & + & - \\
\hline Omphalodes scorpioides (Haenke) Schrank & $\mathrm{T}$ & MsT & $\mathrm{HeSc}$ & $\operatorname{MgTr}$ & Ms & SilRu & RLD & + & - \\
\hline Pulmonaria obscura Dumort. & $\mathrm{HKr}$ & MsT & $\mathrm{HeSc}$ & $\mathrm{MsTr}$ & Ms & Sil & - & + & - \\
\hline Symphytum officinale L. & $\mathrm{HKr}$ & $\mathrm{MgT}$ & $\mathrm{ScHe}$ & $\operatorname{MgTr}$ & $\mathrm{MsHg}$ & PalPr & - & + & + \\
\hline \multicolumn{10}{|l|}{ Brassicaceae } \\
\hline Alliaria petiolata (Bieb.) Cavara et Grande & $\mathrm{HKr}$ & MgT & $\mathrm{HeSc}$ & MsTr & XMs & RuSil & - & + & - \\
\hline Alyssum desertorum Stapf. & $\mathrm{T}$ & $\mathrm{MgT}$ & $\mathrm{He}$ & MsTr & MsX & RuSt & - & + & + \\
\hline Arabidopsis thaliana (L.) Heynh. & $\mathrm{HKr}$ & $\mathrm{MgT}$ & $\mathrm{He}$ & $\mathrm{Og} \operatorname{Tr}$ & XMs & PsRu & Adv & + & + \\
\hline A. toxophylla (Bieb.) N.Busch. & $\mathrm{HKr}$ & $\mathrm{MgT}$ & $\mathrm{He}$ & AlkMgTr & $\mathrm{HgMs}$ & $\mathrm{HPr}$ & - & - & + \\
\hline Arabis pendula $\mathrm{L}$. & $\mathrm{HKr}$ & $\mathrm{MgT}$ & $\mathrm{HeSc}$ & $\operatorname{MgTr}$ & Ms & RuSil & - & + & - \\
\hline Barbarea stricta Andrz. & $\mathrm{THKr}$ & MgT & $\mathrm{ScHe}$ & $\operatorname{MgTr}$ & $\mathrm{HgMs}$ & PalPr & - & + & - \\
\hline Barbarea vulgaris R.Br. & $\mathrm{HKr}$ & MsT & $\mathrm{ScHe}$ & $\operatorname{MsMgTr}$ & Ms & $\operatorname{PrRu}$ & - & - & + \\
\hline Berteroa incana (L.) DC. & $\mathrm{HKr}$ & $\mathrm{MgT}$ & $\mathrm{ScHe}$ & Og-MgTr & $\mathrm{XMs}$ & $\mathrm{Ru}$ & - & + & + \\
\hline Brassica campestris L. & $\mathrm{T}$ & MsT & $\mathrm{ScHe}$ & MsTr & $\mathrm{XMs}$ & $\mathrm{Ru}$ & $\mathrm{Adv}$ & - & + \\
\hline Bunias orientalis $\mathrm{L}$. & $\mathrm{THKr}$ & MsT & $\mathrm{ScHe}$ & OgMgTr & $\mathrm{XMs}$ & $\mathrm{Ru}$ & Adv & + & + \\
\hline Camelina microcarpa Andrcz. & $\mathrm{T}$ & MsT & $\mathrm{He}$ & $\mathrm{MsTr}$ & $\mathrm{XMs}$ & SilRu & Adv & + & + \\
\hline Capsella bursa-pastoris (L.) Medik. & $\mathrm{T}$ & MsT & $\mathrm{He}$ & $\operatorname{Ms} T r$ & XMs & $\mathrm{Ru}$ & Adv & + & + \\
\hline Cardamine amara $\mathrm{L}$. & $\mathrm{HKr}$ & MsT & $\mathrm{ScHe}$ & $\mathrm{MsTr}$ & $\mathrm{MsHg}$ & SilPal & - & + & - \\
\hline C. impatiens L. & $\mathrm{THKr}$ & $\mathrm{MgT}$ & $\mathrm{HeSc}$ & $\mathrm{MsTr}$ & $\mathrm{HgMs}$ & PalSil & - & + & - \\
\hline C. parviflora $\mathrm{L}$. & $\mathrm{T}$ & $\mathrm{MgT}$ & $\mathrm{ScHe}$ & $\mathrm{MsTr}$ & $\mathrm{HgMs}$ & PalPsPr & - & + & + \\
\hline Cardaria draba (L.) Desv. & G & MgT & $\mathrm{He}$ & $\operatorname{MsTr}$ & MsX & $\mathrm{Ru}$ & Adv & + & + \\
\hline Chorispora tenella (Pall.) DC. & $\mathrm{T}$ & MgT & $\mathrm{He}$ & MsTr & MsX & $\mathrm{Ru}$ & $\mathrm{Adv}$ & + & + \\
\hline Descurainia sophia (L.) Webb et Plantl & $\mathrm{T}$ & $\mathrm{MgT}$ & $\mathrm{He}$ & $\mathrm{MsTr}$ & $\mathrm{XMs}$ & $\mathrm{Ru}$ & Adv & + & + \\
\hline Diplotaxis muralis (L.) DC. & $\mathrm{THKr}$ & MsT & $\mathrm{He}$ & $\operatorname{MsTr}$ & MsX & $\mathrm{PtRu}$ & Adv & + & + \\
\hline D. tenuifolia (L.) DC. & $\mathrm{HKrCh}$ & MsT & $\mathrm{He}$ & MsTr & MsX & $\mathrm{Ru}$ & $\mathrm{Adv}$ & - & + \\
\hline Draba nemorosa $\mathrm{L}$. & $\mathrm{T}$ & MsT & $\mathrm{He}$ & $\operatorname{MsTr}$ & $\mathrm{XMs}$ & $\mathrm{Ru}$ & - & + & + \\
\hline Erophila verna (L.) Bess. & $\mathrm{T}$ & $\mathrm{MgT}$ & $\mathrm{He}$ & OgMsTr & $\mathrm{XMs}$ & RuPsSt & - & + & + \\
\hline Erysimum cheiranthoides L. & $\mathrm{HKr}$ & MT & $\mathrm{He}$ & $\mathrm{MsTr}$ & Ms & $\mathrm{Ru}$ & Adv & + & + \\
\hline E. diffusum Ehrh. & $\mathrm{HKr}$ & $\mathrm{MgT}$ & $\mathrm{He}$ & $\mathrm{MsTr}$ & X & RuSt & - & - & + \\
\hline E. strictum P. Gaertn. & $\mathrm{HKr}$ & MgT & $\mathrm{ScHe}$ & $\mathrm{MsTr}$ & MsX & RuSt & - & + & - \\
\hline Euclidium syriacum (L.) R.Br. & $\mathrm{T}$ & MgT & $\mathrm{He}$ & $\operatorname{MsTr}$ & X & $\mathrm{Ru}$ & Adv & - & + \\
\hline Hesperis sibirica $\mathrm{L}$. & $\mathrm{HKr}$ & $\mathrm{MgT}$ & $\mathrm{HeSc}$ & $\mathrm{MsMgTr}$ & Ms & Sil & $\mathrm{Adv}$ & + & - \\
\hline Lepidium latifolium $\mathrm{L}$. & $\mathrm{HKr}$ & MsT & $\mathrm{He}$ & AlkMsTr & $\mathrm{XMs}$ & PrH & - & + & + \\
\hline L. ruderale L. & $\mathrm{THKr}$ & MT & $\mathrm{He}$ & AlkMsTr & MsX & $\mathrm{Ru}$ & Adv & - & + \\
\hline Rorippa amphibia (L.) Bess. & G & $\mathrm{MgT}$ & $\mathrm{HeSc}$ & $\operatorname{MgTr}$ & HyHg & AqPal & - & + & - \\
\hline Sisymbrium loeselii L. & $\mathrm{THKr}$ & $\mathrm{MgT}$ & $\mathrm{He}$ & OgMsTr & MsX & $\mathrm{Ru}$ & $\mathrm{Adv}$ & + & + \\
\hline S. officinale (L.) Scop. & $\mathrm{THKr}$ & MsT & $\mathrm{He}$ & $\mathrm{MsTr}$ & MsX & $\mathrm{Ru}$ & - & - & + \\
\hline Thlaspi arvense L. & $\mathrm{T}$ & MgT & $\mathrm{He}$ & MsTr & MsX & $\mathrm{Ru}$ & Adv & + & + \\
\hline T. perfoliatum $\mathrm{L}$. & $\mathrm{T}$ & MsT & $\mathrm{He}$ & $\mathrm{Ms} T r$ & $\mathrm{XMs}$ & PrStRu & Adv & - & + \\
\hline \multicolumn{10}{|l|}{$\begin{array}{l}\text { Turritis glabra L. } \\
\qquad \text { Caesalpiniaceae }\end{array}$} \\
\hline \multicolumn{9}{|l|}{ Callitrichaceae } & - \\
\hline Callitriche cophocarpa Sendtner & $\mathrm{T}$ & MT & $\mathrm{ScHe}$ & MsMgTr & Hy & PalAq & - & + & - \\
\hline C. palustris $\mathrm{L}$. & $\mathrm{T}$ & EuT & $\mathrm{ScHe}$ & $\operatorname{MsMgTr}$ & Hy & AqPal & RLD & + & - \\
\hline \multicolumn{10}{|l|}{ Campanulaceae } \\
\hline Campanula glomerata L. & $\mathrm{HKr}$ & MsT & $\mathrm{ScHe}$ & MsTr & $\mathrm{XMs}$ & StPrSil & RLD & + & + \\
\hline C. patula $\mathrm{L}$. & $\mathrm{HKr}$ & MsT & $\mathrm{ScHe}$ & $\mathrm{MsTr}$ & $\mathrm{XMs}$ & SilPr & - & + & - \\
\hline C. persicifolia $\mathrm{L}$. & $\mathrm{HKr}$ & MsT & $\mathrm{HeSc}$ & $\mathrm{MsTr}$ & Ms & PsSil & RLD & + & - \\
\hline C. rapunculoides $\mathrm{L}$. & $\mathrm{HKr}$ & MsT & $\mathrm{ScHe}$ & MsTr & $\mathrm{XMs}$ & StPrSil & - & + & + \\
\hline C. rapunculus $\mathrm{L}$. & $\mathrm{HKr}$ & $\mathrm{MgT}$ & $\mathrm{ScHe}$ & MsTr & Ms & $\mathrm{SMnPr}$ & - & + & + \\
\hline C. trachelium $\mathrm{L}$. & $\mathrm{HKr}$ & MsT & $\mathrm{HeSc}$ & MsTr & Ms & SMnSil & RLD & + & - \\
\hline $\begin{array}{l}\text { Cannabaceae } \\
\text { Humulus lupulus L. }\end{array}$ & $\mathrm{G}$ & MsT & $\mathrm{HeSc}$ & $\operatorname{MsTr}$ & $\mathrm{HgMs}$ & RuSil & - & + & + \\
\hline
\end{tabular}




\begin{tabular}{|c|c|c|c|c|c|c|c|c|c|}
\hline 1 & 2 & 3 & 4 & 5 & 6 & 7 & 8 & 9 & 10 \\
\hline \multicolumn{10}{|l|}{ Caprifoliaceae } \\
\hline Lonicera tatarica $\mathrm{L}$. & $\mathrm{nPh}$ & $\mathrm{MgT}$ & $\mathrm{ScHe}$ & MsTr & MsX & $\mathrm{SMnCu}$ & Adv & + & + \\
\hline Sambucus nigra L. & $\mathrm{nPh}$ & $\mathrm{MgT}$ & $\mathrm{ScHe}$ & $\operatorname{MgMsTr}$ & Ms & RuSil & - & + & + \\
\hline Viburnum opulus L. & $\mathrm{nPh}$ & MgT & $\mathrm{ScHe}$ & MgMsTr & Ms & PtSil & - & + & - \\
\hline \multicolumn{10}{|l|}{ Caryophyllaceae } \\
\hline Cerastium holosteoides Fries. & $\mathrm{HKr}$ & EuT & $\mathrm{ScHe}$ & MgMsTr & Ms & $\mathrm{SMnPr}$ & - & + & - \\
\hline C. nemorale Bieb. & $\mathrm{T}$ & $\mathrm{MgT}$ & $\mathrm{ScHe}$ & MsTr & Ms & PrSil & - & + & - \\
\hline Coccyganthe flos-cuculi (L.) Four. & $\mathrm{HKr}$ & $\mathrm{MgT}$ & $\mathrm{ScHe}$ & $\operatorname{Ms} T r$ & $\mathrm{MsHg}$ & SMnPalPr & RLD & + & + \\
\hline Cucubalus baccifer $\mathrm{L}$. & $\mathrm{HKr}$ & $\mathrm{MgT}$ & $\mathrm{HeSc}$ & $\operatorname{MgTr}$ & $\mathrm{HgMs}$ & PrSil & - & + & - \\
\hline Dianthus platyodon Klokov & $\mathrm{HKr}$ & MsT & $\mathrm{He}$ & OgTr & MsX & SilPs & - & + & + \\
\hline Elisanthe noctiflora (L.) Rupr. & $\mathrm{HKr}$ & $\mathrm{MgT}$ & $\mathrm{ScHe}$ & MsTr & $\mathrm{XMs}$ & $\mathrm{SMnPr}$ & - & + & - \\
\hline Herniaria glabra L. & $\mathrm{T}$ & MsT & $\mathrm{He}$ & $\mathrm{MsTr}$ & MsX & RuSt & - & + & + \\
\hline Holosteum umbellatum $\mathrm{L}$. & $\mathrm{T}$ & $\mathrm{MgT}$ & $\mathrm{He}$ & OgMsTr & $\mathrm{XMs}$ & RuPtSt & - & + & + \\
\hline Melandrium album (Mill.) Garcke & $\mathrm{HKr}$ & $\mathrm{MgT}$ & $\mathrm{ScHe}$ & $\mathrm{MsTr}$ & MsX & RuSMnPr & - & + & + \\
\hline Moeringia trinervia (L.) Clairv. & $\mathrm{THKr}$ & $\mathrm{MgT}$ & $\mathrm{HeSc}$ & OgMsTr & $\mathrm{HgMs}$ & Sil & - & + & + \\
\hline Myosoton aquaticum (L.) Moench & $\mathrm{HKr}$ & $\mathrm{MgT}$ & $\mathrm{ScHe}$ & Og-MsTr & $\mathrm{HgMs}$ & PalPr & - & + & + \\
\hline Oberna behen (L.) Ikonn. & $\mathrm{HKrCh}$ & $\mathrm{MgT}$ & $\mathrm{ScHe}$ & $\mathrm{MsTr}$ & $\mathrm{XMs}$ & RuSMnPr & - & + & + \\
\hline Psammophiliella muralis (L.) Ikonn. & $\mathrm{T}$ & MgT & $\mathrm{He}$ & MsTr & Ms & RuPrSt & - & - & + \\
\hline Sagina procumbens L. & $\mathrm{ChHKr}$ & MsT & $\mathrm{ScHe}$ & $\operatorname{MsMgTr}$ & $\mathrm{HgMs}$ & $\mathrm{SMnPr}$ & - & + & + \\
\hline Saponaria officinalis L. & $\mathrm{HKr}$ & $\mathrm{MgT}$ & $\mathrm{ScHe}$ & OgMsTr & Ms & RuSMnPr & $\mathrm{Adv}$ & + & + \\
\hline Silene chlorantha (Willd.) Ehrh. & $\mathrm{HKr}$ & MgT & $\mathrm{ScHe}$ & OgMsTr & XMs & PsSilSt & - & + & + \\
\hline S. dichotoma Ehrh. & $\mathrm{THKr}$ & MsT & $\mathrm{He}$ & $\mathrm{MsTr}$ & $\mathrm{XMs}$ & $\mathrm{StRu}$ & - & - & + \\
\hline Stellaria graminea L. & $\mathrm{HKr}$ & MsT & $\mathrm{ScHe}$ & OgMsTr & Ms & $\mathrm{SMnPr}$ & - & + & - \\
\hline S. holostea L. & $\mathrm{ChHKr}$ & $\mathrm{MgT}$ & $\mathrm{HeSc}$ & $\mathrm{MsTr}$ & Ms & Sil & - & + & - \\
\hline S. media (L.) Vill. & $\mathrm{THKr}$ & MsT & $\mathrm{ScHe}$ & $\operatorname{MsMgTr}$ & $\mathrm{HgMs}$ & SilRu & - & + & + \\
\hline S. neglecta Weihe & $\mathrm{THKr}$ & $\mathrm{MgT}$ & $\mathrm{ScHe}$ & $\operatorname{MgMsTr}$ & $\mathrm{HgMs}$ & RuSil & - & + & - \\
\hline S. nemorum L. & $\mathrm{HKr}$ & MsT & $\mathrm{HeSc}$ & $\mathrm{MsTr}$ & HgMs & Sil & RLD & + & - \\
\hline S. palustris Retz. & $\mathrm{HKr}$ & MsT & $\mathrm{He}$ & $\operatorname{MsMgTr}$ & $\mathrm{Hg}$ & PalPr & RLD & + & - \\
\hline \multicolumn{10}{|l|}{ Celastraceae } \\
\hline Euonymus europaea L. & $\mathrm{nPh}$ & $\mathrm{MgT}$ & $\mathrm{HeSc}$ & MsTr & Ms & SMnSil & - & + & - \\
\hline E. verrucosa Scop. & $\mathrm{nPh}$ & $\mathrm{MgT}$ & $\mathrm{HeSc}$ & MsTr & Ms & SMnSil & - & + & - \\
\hline \multicolumn{10}{|l|}{ Ceratophyllaceae } \\
\hline Ceratophyllum demersum $\mathrm{L}$. & $\mathrm{HKr}$ & EuT & $\mathrm{Sc}$ & AlkMgTr & Hy & $\mathrm{Aq}$ & - & + & + \\
\hline C. pentacanthum Haynald & $\mathrm{HKr}$ & MsT & $\mathrm{Sc}$ & $\operatorname{MgTr}$ & Hy & $\mathrm{Aq}$ & RLD & + & - \\
\hline C. submersum $\mathrm{L}$. & $\mathrm{HKr}$ & $\mathrm{MgT}$ & $\mathrm{Sc}$ & $\operatorname{MgTr}$ & Hy & $\mathrm{Aq}$ & - & + & - \\
\hline C. tanaiticum Sapjeg. & $\mathrm{HKr}$ & MsT & $\mathrm{HeSc}$ & $\operatorname{MgTr}$ & Hy & $\mathrm{Aq}$ & RLD & + & - \\
\hline \multicolumn{10}{|l|}{ Chenopodiaceae } \\
\hline Atriplex patula L. & $\mathrm{T}$ & $\mathrm{MgT}$ & $\mathrm{He}$ & MsTr & XMs & $\mathrm{Ru}$ & - & - & + \\
\hline A. prostrata Boucher & $\mathrm{T}$ & MsT & $\mathrm{ScHe}$ & AlkMsTr & XMs & RuHalPr & Adv & + & + \\
\hline A. rosea $\mathrm{L}$. & $\mathrm{T}$ & $\mathrm{MgT}$ & $\mathrm{He}$ & $\mathrm{MsTr}$ & XMs & $\mathrm{CrRu}$ & - & - & + \\
\hline A. sagittata Borkh & $\mathrm{T}$ & MgT & $\mathrm{He}$ & AlkMsTr & $\mathrm{MsHg}$ & RuHalPr & $\mathrm{Adv}$ & + & + \\
\hline A. tatarica $\mathrm{L}$. & $\mathrm{T}$ & $\mathrm{MgT}$ & $\mathrm{He}$ & MsTr & MsX & RuHal & Adv & + & + \\
\hline Ceratocarpus arenarius L. & $\mathrm{T}$ & MgT & $\mathrm{ScHe}$ & OgMsTr & MsX & RuStPs & - & - & + \\
\hline Chenopodium album $\mathrm{L}$. & $\mathrm{T}$ & EuT & $\mathrm{ScHe}$ & MsTr & MsX & $\mathrm{Ru}$ & - & + & + \\
\hline C. glaucum $\mathrm{L}$. & $\mathrm{T}$ & $\mathrm{MgT}$ & $\mathrm{He}$ & AlkTr & $\mathrm{MsHg}$ & PrRuHal & - & + & + \\
\hline C. hybridum L. & $\mathrm{T}$ & $\mathrm{MgT}$ & $\mathrm{HeSc}$ & MsTr & $\mathrm{XMs}$ & SilRu & Adv & + & - \\
\hline C. polyspermum $\mathrm{L}$. & $\mathrm{T}$ & MsT & $\mathrm{HeSc}$ & OgMsTr & $\mathrm{XMs}$ & RuSMnPs & $\mathrm{Adv}$ & + & + \\
\hline C. rubrum $\mathrm{L}$. & $\mathrm{T}$ & $\mathrm{MgT}$ & $\mathrm{ScHe}$ & $\mathrm{MsTr}$ & $\mathrm{HgMs}$ & RuPs & Adv & + & + \\
\hline C. urbicum $\mathrm{L}$. & $\mathrm{T}$ & MsT & $\mathrm{He}$ & $\operatorname{Ms} T r$ & Ms & $\mathrm{Ru}$ & - & + & + \\
\hline \multicolumn{10}{|l|}{ Clusiaceae } \\
\hline Hypericum hirsutum $\mathrm{L}$. & $\mathrm{HKr}$ & $\mathrm{MgT}$ & $\mathrm{HeSc}$ & $\operatorname{MgTr}$ & XMs & SMnSil & - & + & - \\
\hline H. perforatum $\mathrm{L}$. & $\mathrm{HKr}$ & MgT & $\mathrm{ScHe}$ & OgMsTr & Ms & $\mathrm{SMnPr}$ & - & + & + \\
\hline Convolvulaceae & & & & & & & & & \\
\hline Calystegia sepium (L.) R. Br. & $\mathrm{HKr}$ & EuT & $\mathrm{ScHe}$ & $\operatorname{MsTr}$ & $\mathrm{MsHg}$ & PrPal & - & + & - \\
\hline Convolvulus arvensis $\mathrm{L}$. & $\mathrm{G}$ & EuT & $\mathrm{ScHe}$ & MsTr & MsX & $\mathrm{Ru}$ & - & + & + \\
\hline Cornaceae & & & & & & & & & \\
\hline Swida sanquinea (L.) Opiz. & $\mathrm{Ph}$ & MsT & $\mathrm{HeSc}$ & MsTr & Ms & Sil & - & + & - \\
\hline Corylaceae & & & & & & & & & \\
\hline $\begin{array}{l}\text { Corylus avellana L. } \\
\text { Cuscutaceae }\end{array}$ & $\mathrm{Ph}$ & $\mathrm{MgT}$ & $\mathrm{HeSc}$ & MsTr & Ms & Sil & - & + & - \\
\hline Cuscuta europaea L. & $\mathrm{T}$ & $\mathrm{MgT}$ & $\mathrm{He}$ & Par & HgMs & PrSil & - & + & + \\
\hline Cuscuta lupuliformis Krock. & $\mathrm{T}$ & $\mathrm{MgT}$ & $\mathrm{He}$ & Par & Ms & SilRu & - & + & + \\
\hline Dipsacaceae & & & & & & & & & \\
\hline Dipsacus strigosus Willd. ex Roem & $\mathrm{HKr}$ & $\mathrm{MgT}$ & $\mathrm{HeSc}$ & $\operatorname{MgTr}$ & $\mathrm{HgMs}$ & RuSil & - & + & - \\
\hline Knautia arvensis (L.) Coult. & $\mathrm{HKr}$ & $\mathrm{MgT}$ & $\mathrm{He}$ & MsTr & $\mathrm{XMs}$ & PrSMn & - & + & + \\
\hline Scabiosa ucrainica L. & $\mathrm{HKr}$ & MsT & $\mathrm{He}$ & $\mathrm{Og} \operatorname{Tr}$ & MsX & StPtPs & - & + & - \\
\hline Elaeagnaceae & & & & & & & & & \\
\hline Elaeagnus angustifolia L. & $\mathrm{Ph}$ & $\mathrm{MgT}$ & $\mathrm{He}$ & AlkMsTr & Ms & SMnPrRu & Adv & + & + \\
\hline Elatinaceae & & & & & & & & & \\
\hline Elatine alsinastrum L. & $\mathrm{T}$ & $\mathrm{MgT}$ & $\mathrm{ScHe}$ & MsTr & Hd & PalAq & RLD & + & - \\
\hline Euphorbiaceae & & & & & & & & & \\
\hline Euphorbia kaleniczenkoi Czern. & $\mathrm{HKr}$ & MsT & $\mathrm{He}$ & $\operatorname{MgTr}$ & MsX & RuStPr & - & + & + \\
\hline E. palustris L. & $\mathrm{HKr}$ & MsT & $\mathrm{He}$ & $\operatorname{MgTr}$ & $\mathrm{MsHg}$ & PalPr & - & + & + \\
\hline E. seguieriana Nesk. & $\mathrm{HKr}$ & $\mathrm{MgT}$ & $\mathrm{He}$ & $\mathrm{MsOgTr}$ & MsX & PtStPs & - & + & + \\
\hline E. semivillosa Prokh. & $\mathrm{HKr}$ & $\mathrm{MgT}$ & $\mathrm{ScHe}$ & MsTr & Ms & PrSMn & - & + & + \\
\hline E. virgata Wald. et Kit. & $\mathrm{HKr}$ & MsT & $\mathrm{ScHe}$ & $\operatorname{Ms} T r$ & Ms & $\mathrm{RuPr}$ & - & + & + \\
\hline
\end{tabular}




\begin{tabular}{|c|c|c|c|c|c|c|c|c|c|}
\hline 1 & 2 & 3 & 4 & 5 & 6 & 7 & 8 & 9 & 10 \\
\hline \multicolumn{10}{|l|}{ Fabaceae } \\
\hline Amorpha fruticosa $\mathrm{L}$. & $\mathrm{nPh}$ & MsT & $\mathrm{ScHe}$ & OgMsTr & $\mathrm{HgMs}$ & CuRuSil & Adv & + & + \\
\hline Astragalus ausriacus Jacq. & $\mathrm{HKr}$ & MgT & $\mathrm{ScHe}$ & OgMsTr & $\mathrm{X}$ & PtSt & - & - & + \\
\hline A. cicer $\mathrm{L}$. & $\mathrm{HKr}$ & $\mathrm{MgT}$ & $\mathrm{ScHe}$ & $\mathrm{MsTr}$ & $\mathrm{XMs}$ & RuStPr & - & + & + \\
\hline A. glycyphyllos L. & $\mathrm{HKr}$ & $\mathrm{MgT}$ & $\mathrm{HeSc}$ & $\operatorname{MgTr}$ & Ms & Sil & - & + & - \\
\hline Caragana arborescens $\mathrm{L}$. & $\mathrm{Ph}$ & MsT & $\mathrm{He}$ & MsTr & MsX & CuSil & - & + & - \\
\hline Chamecytisus lindemanii (V.Krecz.) Klaskova & $\mathrm{Ch}$ & MsT & $\mathrm{ScHe}$ & $\operatorname{MsTr}$ & XMs & SMnSt & ERL, RLD & + & + \\
\hline Genista tinctoria L. & $\mathrm{nPh}$ & MsT & $\mathrm{ScHe}$ & $\mathrm{MsOgTr}$ & $\mathrm{XMs}$ & PrPsSMn & - & + & - \\
\hline Lathyrus incurvus (Roth) Roth & $\mathrm{G}$ & $\operatorname{MgT}$ & $\mathrm{He}$ & AlkMsTr & XMs & HalPr & RLD & + & + \\
\hline L. palustris $\mathrm{L}$. & $\mathrm{HKr}$ & MgT & $\mathrm{He}$ & MsMgT & $\mathrm{MsHg}$ & PalPr & RLD & + & - \\
\hline L. pratensis $\mathrm{L}$. & $\mathrm{GHKr}$ & $\mathrm{MgT}$ & $\mathrm{He}$ & $\operatorname{MgTr}$ & Ms & $\mathrm{SMnPr}$ & - & - & + \\
\hline L. sylvestris L. & $\mathrm{HKr}$ & MgT & $\mathrm{ScHe}$ & MsTr & Ms & SilSMn & - & + & - \\
\hline L. tuberosus L. & $\mathrm{G}$ & $\mathrm{MgT}$ & $\mathrm{He}$ & MsMgTr & MsX & RuPrSt & Adv & + & + \\
\hline L. vernus (L.) Bernh. & G & MsT & $\mathrm{HeSc}$ & MsTr & Ms & SilSMn & RLD & + & - \\
\hline Lotus praetermissus Kuprian. & $\mathrm{T}$ & $\mathrm{MgT}$ & $\mathrm{He}$ & $\mathrm{OgTr}$ & Ms & Ps & - & + & + \\
\hline L. ucrainicus Klokov & $\mathrm{HKr}$ & MsT & $\mathrm{He}$ & MgMsTr & XMs & $\mathrm{StPr}$ & - & + & + \\
\hline Medicago lupulina $\mathrm{L}$. & $\mathrm{THKr}$ & MgT & $\mathrm{He}$ & MsMgTr & Ms & RuSMnPr & - & + & + \\
\hline M. romanica Prod. & $\mathrm{HKr}$ & MgT & $\mathrm{He}$ & MsTr & MsX & PrPtSt & - & + & + \\
\hline Melilotus albus Medik. & $\mathrm{HKr}$ & MgT & $\mathrm{He}$ & MsTr & MsX & RuPrSt & - & - & + \\
\hline M. officinalis (L.) Pall. & $\mathrm{HKr}$ & EuT & $\mathrm{He}$ & $\mathrm{Ms} \operatorname{Tr}$ & XMs & RuStPr & - & - & + \\
\hline Ononis arvensis L. & $\mathrm{HKr}$ & $\mathrm{MgT}$ & $\mathrm{He}$ & AlkMgTr & Ms & HalPr & - & - & + \\
\hline Robinia pseudoacacia L. & $\mathrm{Ph}$ & MsT & $\mathrm{He}$ & Og-MgTr & X-Ms & CuSil & Adv & + & + \\
\hline Securigera varia $\mathrm{L}$. & $\mathrm{HKr}$ & MgT & $\mathrm{He}$ & MsTr & XMs & $\mathrm{StSMnPr}$ & - & + & + \\
\hline Trifolium alpestre $\mathrm{L}$. & $\mathrm{HKr}$ & $\mathrm{MgT}$ & $\mathrm{He}$ & $\operatorname{MgTr}$ & $\mathrm{XMs}$ & SilStPr & - & + & + \\
\hline T. ambiguum Bieb. & $\mathrm{HKr}$ & $\mathrm{MgT}$ & $\mathrm{He}$ & $\operatorname{MgTr}$ & Ms & RuHalPr & - & - & + \\
\hline T. aureum L. & $\mathrm{T}$ & $\mathrm{MgT}$ & $\mathrm{ScHe}$ & $\mathrm{MsTr}$ & Ms & $\mathrm{SMnPr}$ & - & + & + \\
\hline T. borysthenicum Grun. & $\mathrm{HKr}$ & MgT & $\mathrm{He}$ & AlkMsTr & Ms & HalPr & - & - & + \\
\hline T. fragiferum $\mathrm{L}$. & $\mathrm{HKr}$ & $\mathrm{MgT}$ & $\mathrm{He}$ & AlkTr & $\mathrm{HgMs}$ & HalPr & - & - & + \\
\hline T. medium L. & $\mathrm{G}$ & MgT & $\mathrm{ScHe}$ & $\operatorname{MgTr}$ & Ms & SilPr & - & + & - \\
\hline T. montanum $\mathrm{L}$. & $\mathrm{HKr}$ & $\mathrm{MgT}$ & $\mathrm{HeSc}$ & $\operatorname{MgTr}$ & $\mathrm{XMs}$ & $\mathrm{SMnPr}$ & - & + & - \\
\hline T. pratense $\mathrm{L}$. & $\mathrm{HKr}$ & $\mathrm{MgT}$ & $\mathrm{He}$ & $\operatorname{MgTr}$ & $\mathrm{HgMs}$ & RuSMnPr & - & + & + \\
\hline T. repens L. & $\mathrm{HKr}$ & MT & $\mathrm{He}$ & $\operatorname{MgTr}$ & $\mathrm{HgMs}$ & $\mathrm{RuPr}$ & - & + & + \\
\hline Trigonella caerulea (L.) Ser. & $\mathrm{T}$ & MgT & $\mathrm{He}$ & MsTr & Ms & $\mathrm{RuPr}$ & Adv & - & + \\
\hline Vicia angustifolia Reichard & $\mathrm{T}$ & $\mathrm{MgT}$ & $\mathrm{He}$ & $\operatorname{MsMgTr}$ & $\mathrm{XMs}$ & $\mathrm{RuPr}$ & Adv & - & + \\
\hline V. cracca $\mathrm{L}$. & $\mathrm{HKr}$ & MsT & $\mathrm{He}$ & $\mathrm{MsTr}$ & $\mathrm{HgMs}$ & PrSt & - & + & + \\
\hline V. dasycarpa Ten. & $\mathrm{HKr}$ & $\mathrm{MgT}$ & $\mathrm{He}$ & $\mathrm{MsTr}$ & $\mathrm{XMs}$ & SilPr & - & + & + \\
\hline$V$. grandiflora Scop. & $\mathrm{HKr}$ & MsT & $\mathrm{He}$ & MsTr & Ms & $\mathrm{SMnPr}$ & - & + & + \\
\hline V. hirsuta (L.) S.F.Grag & $\mathrm{T}$ & $\operatorname{MgT}$ & $\mathrm{ScHe}$ & $\operatorname{MsTr}$ & MsX & RuSilSt & Adv & + & + \\
\hline V. picta Fisch. et Mey. & $\mathrm{HKr}$ & MgT & $\mathrm{ScHe}$ & $\operatorname{MgTr}$ & $\mathrm{HgMs}$ & $\operatorname{Pr}$ & Adv & + & + \\
\hline V.pisiformis $\mathrm{L}$. & $\mathrm{HKr}$ & $\mathrm{MgT}$ & $\mathrm{He}$ & $\mathrm{MsTr}$ & Ms & Sil & - & + & - \\
\hline V. sepium $\mathrm{L}$. & $\mathrm{HKr}$ & MsT & $\mathrm{He}$ & $\operatorname{MgTr}$ & Ms & $\mathrm{SMnPr}$ & - & + & + \\
\hline V. tenuifolia Roth. & $\mathrm{HKr}$ & MsT & $\mathrm{He}$ & $\operatorname{MgTr}$ & MsX & SMnStPr & - & + & + \\
\hline V. tetrasperma (L.) Schreb. & $\mathrm{T}$ & MsT & $\mathrm{ScHe}$ & $\operatorname{MgTr}$ & $\mathrm{XMs}$ & RuSMnPr & $\mathrm{Adv}$ & + & + \\
\hline$V$. villosa Roth. & $\mathrm{HKr}$ & $\operatorname{MgT}$ & $\mathrm{ScHe}$ & $\operatorname{MgTr}$ & $\mathrm{XMs}$ & RuSMnPr & - & + & + \\
\hline \multicolumn{10}{|l|}{ Fagaceae } \\
\hline Quercus robur $\mathrm{L}$. & $\mathrm{Ph}$ & MsT & $\mathrm{ScHe}$ & OgMsMgTr & Ms & Sil & - & + & - \\
\hline \multicolumn{10}{|l|}{ Fumariaceae } \\
\hline Corydalis cava (L.) Schweigg. et Korte & G & MsT & $\mathrm{ScHe}$ & $\operatorname{MgTr}$ & Ms & Sil & RLD & + & - \\
\hline Corydalis marschalliana Pers. & $\mathrm{G}$ & MgT & $\mathrm{ScHe}$ & $\operatorname{MgTr}$ & Ms & Sil & RLD & + & - \\
\hline Corydalis solida (L.) Clairv. & $\mathrm{G}$ & MsT & $\mathrm{ScHe}$ & $\operatorname{MgTr}$ & Ms & Sil & - & + & - \\
\hline Fumaria officinalis L. & $\mathrm{T}$ & MgT & $\mathrm{He}$ & MsTr & MsX & $\mathrm{Ru}$ & Adv & + & + \\
\hline Fumaria schleicheri Soy.-Willem. & $\mathrm{T}$ & $\mathrm{MgT}$ & $\mathrm{ScHe}$ & $\mathrm{MsTr}$ & MsX & $\mathrm{Ru}$ & Adv & + & + \\
\hline \multicolumn{10}{|l|}{ Gentianaceae } \\
\hline Centaurium erythraea Rafn. & $\mathrm{THKr}$ & $\operatorname{MgT}$ & $\mathrm{ScHe}$ & OgMsTr & XMs & $\mathrm{StPr}$ & - & + & + \\
\hline Centaurium pulchellum (Sw.) Druce & $\mathrm{T}$ & MsT & $\mathrm{He}$ & $\mathrm{MsTr}$ & $\mathrm{HgMs}$ & Pr & - & + & + \\
\hline Gentiana pneumonante L. & $\mathrm{HKr}$ & MsT & $\mathrm{ScHe}$ & $\operatorname{MsTr}$ & $\mathrm{MsHg}$ & SilPalPr & RLD & + & - \\
\hline \multicolumn{10}{|l|}{ Geraniaceae } \\
\hline Erodium cicutarium (L.) L Her. & $\mathrm{T}$ & $\mathrm{MgT}$ & $\mathrm{ScHe}$ & OgMsTr & XMs & SilPtStRu & - & + & - \\
\hline Geranium collinum Steph. & $\mathrm{HKr}$ & MgT & $\mathrm{ScHe}$ & AlkTr & $\mathrm{HgMs}$ & PalHalPr & - & + & + \\
\hline G. divaricatum Ehrh. & $\mathrm{T}$ & MgT & $\mathrm{ScHe}$ & OgMsTr & $\mathrm{XMs}$ & RuSil & - & + & - \\
\hline G. palustre L. & $\mathrm{HKr}$ & $\mathrm{MgT}$ & $\mathrm{ScHe}$ & MsTr & $\mathrm{MsHg}$ & PrPal & RLD & + & - \\
\hline G. pratense L. & $\mathrm{HKr}$ & MsT & $\mathrm{HeSc}$ & $\operatorname{MsTr}$ & $\mathrm{Ms}$ & PrSMn & RLD & + & + \\
\hline G. pusillum L. & $\mathrm{T}$ & $\mathrm{MgT}$ & $\mathrm{He}$ & MsTr & MsX & $\mathrm{Ru}$ & Adv & + & + \\
\hline G. robertianum L. & $\mathrm{T}$ & $\mathrm{MgT}$ & $\mathrm{ScHe}$ & OgMsTr & $\mathrm{XMs}$ & PtPsSil & - & + & - \\
\hline G. sanguineum $\mathrm{L}$. & $\mathrm{G}$ & $\mathrm{MgT}$ & $\mathrm{ScHe}$ & $\operatorname{Og} \operatorname{Tr}$ & $\mathrm{XMs}$ & PsSil & - & + & - \\
\hline Grossulariaceae & & & & & & & & & \\
\hline Ribes aureum Pursh & $\mathrm{nPh}$ & MsT & $\mathrm{ScHe}$ & $\operatorname{MsTr}$ & Ms & SilSMnCu & Adv & + & - \\
\hline Haloragaceae & & & & & & & & & \\
\hline Myriophyllum spicatum L. & $\mathrm{HKr}$ & MsT & $\mathrm{ScHe}$ & $\operatorname{MsTr}$ & Hy & $\mathrm{Aq}$ & - & + & + \\
\hline M. verticillatum $\mathrm{L}$. & $\mathrm{HKr}$ & $\mathrm{MgT}$ & $\mathrm{HeSc}$ & $\operatorname{MsTr}$ & Hy & $\mathrm{Aq}$ & - & + & - \\
\hline Lamiaceae & & & & & & & & & \\
\hline Acinos arvensis (Lam.) Dandy & $\mathrm{T}, \mathrm{HKr}$ & MsT & $\mathrm{ScHe}$ & $\operatorname{MsTr}$ & MsX & RuPtSt & - & + & - \\
\hline Ajuga genevensis L. & G & $\operatorname{MgT}$ & $\mathrm{ScHe}$ & MsTr & $\mathrm{XMs}$ & RuPrSil & - & + & + \\
\hline Ballota nigra $\mathrm{L}$ & $\mathrm{HKr}$ & MsT & $\mathrm{ScHe}$ & $\operatorname{MsTr}$ & Ms & $\mathrm{SilRu}$ & Adv & + & + \\
\hline Betonica officinalis L. & $\mathrm{HKr}$ & $\operatorname{MgT}$ & $\mathrm{ScHe}$ & $\mathrm{Og} \operatorname{Tr}$ & Ms & PrSil & RLD & + & + \\
\hline Chaiturus marrubiastrum (L.) Reichenb. & $\mathrm{THKr}$ & $\mathrm{MgT}$ & $\mathrm{ScHe}$ & MsTr & $\mathrm{XMs}$ & RuPrSMn & - & + & - \\
\hline Clinopodium vulgare $\mathrm{L}$. & $\mathrm{HKr}$ & $\mathrm{MgT}$ & $\mathrm{ScHe}$ & MsTr & $\mathrm{XMs}$ & SilSMn & - & + & - \\
\hline
\end{tabular}




\begin{tabular}{|c|c|c|c|c|c|c|c|c|c|}
\hline 1 & 2 & 3 & 4 & 5 & 6 & 7 & 8 & 9 & 10 \\
\hline Galeopsis. speciosa Mill. & $\mathrm{T}$ & $\operatorname{MgT}$ & $\mathrm{ScHe}$ & MsTr & $\mathrm{XMs}$ & RuSMn & - & - & + \\
\hline G. tetrahit $\mathrm{L}$. & $\mathrm{T}$ & MgT & $\mathrm{ScHe}$ & Og-MsTr & Ms & RuPsSil & - & + & - \\
\hline Glechoma hederacea L. & $\mathrm{HKr}$ & MsT & $\mathrm{HeSc}$ & MsTr & Ms & RuPrSil & - & + & + \\
\hline G. hirsuta Waldst. et Kit. & $\mathrm{HKr}$ & MsT & $\mathrm{HeSc}$ & MsTr & Ms & Sil & - & + & - \\
\hline Lamium amplexicaule $\mathrm{L}$. & $\mathrm{THKr}$ & MsT & $\mathrm{He}$ & OgMsTr & $\mathrm{XMs}$ & $\mathrm{Ru}$ & Adv & + & + \\
\hline L. purpureum L. & $\mathrm{THKr}$ & $\mathrm{MgT}$ & $\mathrm{ScHe}$ & $\mathrm{MsTr}$ & XMs & $\mathrm{Ru}$ & $\mathrm{Adv}$ & + & + \\
\hline Leonurus cardiaca L. & $\mathrm{HKr}$ & MsT & $\mathrm{ScHe}$ & $\operatorname{MgTr}$ & $\mathrm{XMs}$ & $\mathrm{SMnRu}$ & Adv & + & - \\
\hline L. villosus Desf. ex D'Urv. & $\mathrm{HKr}$ & $\mathrm{MgT}$ & $\mathrm{ScHe}$ & MsTr & MsX & $\mathrm{Ru}$ & - & + & + \\
\hline Lycopus europaeus L. & $\mathrm{HKr}$ & MgT & $\mathrm{ScHe}$ & $\operatorname{MgTr}$ & $\mathrm{MsHg}$ & PrPal & - & + & + \\
\hline L. exaltatus L.fil. & $\mathrm{HKr}$ & MsT & $\mathrm{ScHe}$ & $\operatorname{MgTr}$ & $\mathrm{MsHg}$ & PrPal & - & + & + \\
\hline Marrubium praecox Janca & $\mathrm{HKr}$ & $\mathrm{MgT}$ & $\mathrm{He}$ & MsTr & MsX & RuStPt & - & - & + \\
\hline Mentha arvensis L. & $\mathrm{HKr}$ & MgT & $\mathrm{ScHe}$ & MsTr & $\mathrm{HgMs}$ & RuPrSil & - & + & - \\
\hline M. aquatica $\mathrm{L}$. & $\mathrm{HKr}$ & MgT & $\mathrm{ScHe}$ & MsTr & $\mathrm{Hg}$ & PalAq & - & + & + \\
\hline Nepeta cataria L. & $\mathrm{HKr}$ & MsT & $\mathrm{ScHe}$ & MsTr & XMs & RuStSil & Adv & + & + \\
\hline N. pannonica L. & $\mathrm{HKr}$ & MgT & $\mathrm{ScHe}$ & MsTr & MsX & SMnSt & - & + & + \\
\hline Origanum vulgare $\mathrm{L}$. & $\mathrm{HKr}$ & MgT & $\mathrm{ScHe}$ & $\operatorname{MgTr}$ & MsX & StSMnPr & - & + & - \\
\hline Phlomis tuberosa L. & $\mathrm{HKr}$ & MgT & $\mathrm{He}$ & MsTr & MsX & PrSMnSt & - & - & + \\
\hline Prunella vulgaris L. & $\mathrm{HKr}$ & MsT & $\mathrm{He}$ & $\operatorname{MgTr}$ & Ms & RuPrSil & - & + & - \\
\hline Salvia verticillata $\mathrm{L}$. & $\mathrm{HKr}$ & MsT & $\mathrm{ScHe}$ & MsTr & MsX-Ms & PrStRu & - & - & + \\
\hline Scutellaria altissima L. & $\mathrm{HKr}$ & MgT & $\mathrm{HeSc}$ & $\operatorname{MgTr}$ & Ms & Sil & RLD & + & - \\
\hline S. galericulata L. & G & MgT & $\mathrm{ScHe}$ & $\operatorname{MgTr}$ & $\mathrm{Hg}$ & PrPal & - & + & - \\
\hline S. hastifolia L. & $\mathrm{HKr}$ & MsT & $\mathrm{ScHe}$ & $\operatorname{MgTr}$ & $\mathrm{HgMs}$ & PalPr & - & + & - \\
\hline Stachys palustris L. & $\mathrm{G}$ & MT & $\mathrm{ScHe}$ & $\operatorname{MgTr}$ & $\mathrm{Hg}$ & PrPal & - & + & + \\
\hline S. recta L. & $\mathrm{HKr}$ & MgT & $\mathrm{He}$ & $\mathrm{OgTr}$ & MsX & SMnSt & - & + & + \\
\hline S. sylvatica L. & $\mathrm{HKr}$ & $\mathrm{MgT}$ & $\mathrm{HeSc}$ & $\mathrm{MgTr}$ & Ms & Sil & - & + & - \\
\hline Teucrium chamedris $\mathrm{L}$. & $\mathrm{Ch}$ & $\mathrm{MgT}$ & $\mathrm{ScHe}$ & OgMsTr & MsX & SMnPtSt & - & + & + \\
\hline T. scordium $\mathrm{L}$. & $\mathrm{HKr}$ & MsT & $\mathrm{ScHe}$ & $\operatorname{MgTr}$ & HgMs & PalPr & - & + & + \\
\hline \multicolumn{10}{|l|}{$\begin{array}{r}\text { Lentibulariaceae } \\
\end{array}$} \\
\hline $\begin{array}{l}\text { Utricularia vulgaris L. } \\
\text { Limoniaceae }\end{array}$ & \multicolumn{8}{|c|}{ Limoniaceae } & - \\
\hline $\begin{array}{c}\text { Limonium tomentellum (Boiss.) O.Kuntze } \\
\text { Loranthaceae }\end{array}$ & \multicolumn{8}{|c|}{ Loranthaceae } & + \\
\hline Viscum album L. $\quad$ Lythraceae & $\mathrm{nPh}$ & $\mathrm{MgT}$ & $\mathrm{ScHe}$ & Par & $\mathrm{HgMs}$ & Sil & - & + & + \\
\hline Lythrum salicaria L. & $\mathrm{HKr}$ & MsT & $\mathrm{He}$ & $\operatorname{MgTr}$ & $\mathrm{MsHg}$ & PrAqPal & - & + & + \\
\hline L. virgatum $\mathrm{L}$. & $\mathrm{HKr}$ & MsT & $\mathrm{He}$ & $\operatorname{MgTr}$ & $\mathrm{MsHg}$ & $\mathrm{AqPalPr}$ & - & + & + \\
\hline Peplis portula L. & $\mathrm{HKr}$ & $\operatorname{MgT}$ & $\mathrm{ScHe}$ & $\mathrm{MsTr}$ & $\mathrm{MsHg}$ & PsPalPr & - & + & + \\
\hline \multicolumn{10}{|l|}{ Malvaceae } \\
\hline Althaea officinalis L. & $\mathrm{HKr}$ & MgT & $\mathrm{He}$ & AlkMgTr & Ms & HalPalPr & Adv & + & + \\
\hline Lavatera thuringiaca $\mathrm{L}$. & $\mathrm{HKr}$ & MgT & $\mathrm{ScHe}$ & $\operatorname{MgTr}$ & MsX & RuStPr & - & - & + \\
\hline Malva pusilla Smith & $\mathrm{T}$ & MsT & $\mathrm{ScHe}$ & $\mathrm{Ms} \operatorname{Tr}$ & $\mathrm{XMs}$ & $\mathrm{Ru}$ & Adv & + & + \\
\hline \multicolumn{10}{|l|}{ Moraceae } \\
\hline Morus alba L. & $\mathrm{Ph}$ & $\operatorname{MgT}$ & $\mathrm{He}$ & $\operatorname{MsTr}$ & Ms & $\mathrm{CuSilRu}$ & Adv & + & + \\
\hline \multicolumn{10}{|l|}{ Nymphaeaceae } \\
\hline Nuphar lutea (L.) Smith & $\mathrm{HKr}$ & MgT & $\mathrm{He}$ & MsTr & $\mathrm{Pl}$ & $\mathrm{Aq}$ & RLD & + & + \\
\hline Nymphaea alba L. & $\mathrm{HKr}$ & MgT & $\mathrm{ScHe}$ & $\mathrm{MsTr}$ & $\mathrm{Pl}$ & $\mathrm{Aq}$ & RLD & + & - \\
\hline Oleaceae & & & & & & & & & \\
\hline Fraxinus excelsior L. & $\mathrm{Ph}$ & MgT & $\mathrm{ScHe}$ & MsMgTr & Ms & Sil & - & + & + \\
\hline F. lanceolata Borkh. & $\mathrm{Ph}$ & MsT & $\mathrm{ScHe}$ & $\mathrm{MsTr}$ & Ms & CuRuSil & Adv & + & + \\
\hline Ligustrum vulgare L. & $\mathrm{Ph}$ & $\mathrm{MgT}$ & $\mathrm{ScHe}$ & MsTr & $\mathrm{XMs}$ & PtSil & - & + & - \\
\hline Onagraceae & & & & & & & & & \\
\hline Epilobium hirsutum L. & $\mathrm{HKr}$ & MsT & $\mathrm{He}$ & $\operatorname{MgTr}$ & $\mathrm{MsHg}$ & PrPal & - & + & + \\
\hline E. palustre L. & $\mathrm{HKr}$ & MsT & $\mathrm{He}$ & $\mathrm{MgTr}$ & $\mathrm{MsHg}$ & PrPal & RLD & + & - \\
\hline E. parviflorum Schreb. & $\mathrm{HKr}$ & $\mathrm{MgT}$ & $\mathrm{He}$ & MsTr & $\mathrm{Hg}$ & PrPal & - & + & - \\
\hline E. roseum Schreb. & $\mathrm{HKr}$ & MgT & $\mathrm{ScHe}$ & $\operatorname{MgTr}$ & $\mathrm{HgMs}$ & PalPr & - & + & - \\
\hline Papaveraceae & & & & & & & & & \\
\hline Chelidonium majus L. & $\mathrm{HKr}$ & MsT & $\mathrm{HeSc}$ & $\mathrm{MsMgTr}$ & Ms & RuSil & - & + & + \\
\hline Plantaginaceae & & & & & & & & & \\
\hline Plantago cornuti Gousn. & $\mathrm{HKr}$ & MT & $\mathrm{He}$ & AlkTr & Ms & HalPr & - & - & + \\
\hline P. lanceolata $\mathrm{L}$. & $\mathrm{HKr}$ & MT & $\mathrm{He}$ & MsTr & $\mathrm{XMs}$ & PrStSMnRu & - & + & + \\
\hline P. major L. & $\mathrm{HKr}$ & $\operatorname{MgT}$ & $\mathrm{He}$ & $\operatorname{MgTr}$ & Ms & $\mathrm{RuPr}$ & - & + & + \\
\hline P. media L. & $\mathrm{HKr}$ & $\mathrm{MgT}$ & $\mathrm{He}$ & $\operatorname{MgTr}$ & MsX & RuSilPrSt & - & + & + \\
\hline $\begin{array}{l}\text { Polygalaceae } \\
\end{array}$ & & & & & & & & & \\
\hline Polygala comosa Schruhr & $\mathrm{HKr}$ & MsT & $\mathrm{ScHe}$ & MsTr & Ms & PrSMn & - & + & + \\
\hline Polygonaceae & & & & & & & & & \\
\hline Fallopia convolvulus (L.) A.Love & $\mathrm{T}$ & MT & $\mathrm{ScHe}$ & MsTr & XMs & $\mathrm{Ru}$ & Adv & + & + \\
\hline Persicaria amphybia (L.) Delarbre & $\mathrm{HKr}$ & MsT & $\mathrm{ScHe}$ & MsTr & $\mathrm{PlHg}$ & AqPal & - & + & + \\
\hline P. dubia (Stein) Fourr. & $\mathrm{T}$ & MsT & $\mathrm{ScHe}$ & $\operatorname{MgTr}$ & $\mathrm{XMs}$ & PrHal & RLD, ERL & - & + \\
\hline P. hydropiper $\mathrm{L}$. & $\mathrm{T}$ & MsT & $\mathrm{ScHe}$ & MsTr & $\mathrm{HgMs}$ & RuSilPalPr & - & + & - \\
\hline P. lapathifolia (L.) Delarbe & $\mathrm{T}$ & MsT & $\mathrm{He}$ & $\mathrm{Og} \operatorname{Tr}$ & $\mathrm{HgMs}$ & PalPsPr & - & + & + \\
\hline P. maculosa S.F.Gray & $\mathrm{T}$ & $\mathrm{MgT}$ & $\mathrm{He}$ & MsTr & Ms & $\mathrm{RuPr}$ & - & - & + \\
\hline P. minor (Huds.) Opiz & $\mathrm{T}$ & $\mathrm{MgT}$ & $\mathrm{ScHe}$ & OgMsTr & $\mathrm{MsHg}$ & SilPalPr & - & + & + \\
\hline Polygonum scabra (Moench) Moldenke & $\mathrm{T}$ & EuT & $\mathrm{He}$ & $\operatorname{MgTr}$ & $\mathrm{XMs}$ & RuPr & - & + & + \\
\hline$P$. aviculare $\mathrm{L}$. & $\mathrm{T}$ & EuT & $\mathrm{ScHe}$ & MsTr & MsX & $\mathrm{Ru}$ & - & + & + \\
\hline Rumex acetosa L. & $\mathrm{G}$ & $\mathrm{MgT}$ & $\mathrm{He}$ & $\mathrm{MgTr}$ & $\mathrm{XMs}$ & $\mathrm{SMnPr}$ & - & + & - \\
\hline R. acetosella L. & $\mathrm{HKr}$ & MsT & $\mathrm{ScHe}$ & OgMsTr & Ms & RuPrSMnPs & - & + & + \\
\hline R. aquaticus L. & $\mathrm{HKr}$ & $\mathrm{MgT}$ & $\mathrm{ScHe}$ & $\mathrm{MgTr}$ & $\mathrm{MsHg}$ & PalSMn & - & + & - \\
\hline
\end{tabular}




\begin{tabular}{|c|c|c|c|c|c|c|c|c|c|}
\hline 1 & 2 & 3 & 4 & 5 & 6 & 7 & 8 & 9 & 10 \\
\hline R. confertus Willd. & $\mathrm{HKr}$ & MsT & $\mathrm{ScHe}$ & MsTr & $\mathrm{XMs}$ & RuPrSMn & - & + & + \\
\hline R. crispus L. & $\mathrm{HKr}$ & MT & $\mathrm{He}$ & MsTr & Ms & $\mathrm{RuPr}$ & - & + & + \\
\hline R. hydrolapatum Huds. & $\mathrm{HKr}$ & MgT & $\mathrm{He}$ & MsTr & $\mathrm{Hg}$ & AqPal & - & + & - \\
\hline R. longifolius DC. & $\mathrm{HKr}$ & MgT & $\mathrm{He}$ & MsTr & Ms & RuSMnPr & Adv & + & + \\
\hline R. maritimus $\mathrm{L}$. & $\mathrm{HKr}$ & MsT & $\mathrm{He}$ & AlkTr & $\mathrm{HgMs}$ & $\mathrm{HalPr}$ & - & - & + \\
\hline \multicolumn{10}{|l|}{ Primulaceae } \\
\hline Glaux maritima L. & $\mathrm{HKr}$ & MsT & $\mathrm{ScHe}$ & AlkTr & HgMs & PrHal & - & - & + \\
\hline Hottonia palustris L. & $\mathrm{HKr}$ & MsT & $\mathrm{ScHe}$ & $\operatorname{MsTr}$ & Hy & AqPal & RLD & + & - \\
\hline Lysimachia nummularia $\mathrm{L}$. & $\mathrm{HKr}$ & $\mathrm{MgT}$ & $\mathrm{ScHe}$ & $\operatorname{MgTr}$ & HgMs & SilPr & - & + & - \\
\hline L. vulgaris $\mathrm{L}$. & $\mathrm{HKr}$ & MgT & $\mathrm{ScHe}$ & $\operatorname{MgTr}$ & $\mathrm{MsHg}$ & $\mathrm{Pal}$ & - & + & + \\
\hline \multicolumn{10}{|l|}{ Ranunculaceae } \\
\hline Aconitum nemorosum Bieb. ex Reichenb. & $\mathrm{HKr}$ & MsT & $\mathrm{HeSc}$ & $\operatorname{MgTr}$ & Ms & SMn & RLD & + & - \\
\hline A. rogoviczii Wissjul. & $\mathrm{HKr}$ & MsT & $\mathrm{ScHe}$ & MsTr & $\mathrm{XMs}$ & Sil & RLD & + & - \\
\hline Anemone ranunculoides (L.) Holub & $\mathrm{G}$ & $\mathrm{MgT}$ & $\mathrm{HeSc}$ & $\operatorname{MgTr}$ & Ms & Sil & RLD & + & - \\
\hline Batrachium circinatum (Sibth.) Spach & $\mathrm{HKr}$ & MgT & $\mathrm{HeSc}$ & AlkTr & Hy & $\mathrm{Aq}$ & - & + & - \\
\hline B. trichophyllum (Chaix) Bosch & $\mathrm{HKr}$ & MsT & $\mathrm{ScHe}$ & MsTr & Hy & $\mathrm{Aq}$ & - & + & + \\
\hline Caltha palustris L. & $\mathrm{HKr}$ & EuT & $\mathrm{He}$ & $\mathrm{MsTr}$ & $\mathrm{Hg}$ & PrPal & RLD & + & - \\
\hline Ceratocephala testiculata (Crantz) Besser & $\mathrm{T}$ & $\mathrm{MgT}$ & $\mathrm{He}$ & MsTr & $\mathrm{MsX}$ & RuSt & - & + & + \\
\hline Consolida regalis S.F.Gray & $\mathrm{T}$ & MsT & $\mathrm{ScHe}$ & MsTr & $\mathrm{MsX}$ & $\mathrm{SMnRu}$ & Adv & + & + \\
\hline Ficaria verna Huds. & $\mathrm{G}$ & MsT & $\mathrm{HeSc}$ & $\operatorname{MgTr}$ & Ms & Sil & - & + & - \\
\hline Ramunculus auricomus L. & $\mathrm{HKr}$ & $\mathrm{MgT}$ & $\mathrm{ScHe}$ & $\operatorname{MgTr}$ & Ms & SilPr & RLD & + & + \\
\hline R. flammula L. & $\mathrm{HKr}$ & MsT & $\mathrm{He}$ & $\mathrm{MsTr}$ & $\mathrm{HgMs}$ & PalPr & RLD & + & + \\
\hline R. lingua $\mathrm{L}$. & $\mathrm{HKr}$ & $\mathrm{MgT}$ & $\mathrm{ScHe}$ & MsTr & $\mathrm{MsHg}$ & PrPal & RLD & + & - \\
\hline R. pedatus Waldst. et Kit. & $\mathrm{G}$ & MgT & $\mathrm{ScHe}$ & $\operatorname{MgTr}$ & $\mathrm{XMs}$ & StPr & - & + & + \\
\hline R. polyanthemos $\mathrm{L}$. & $\mathrm{HKr}$ & MsT & $\mathrm{ScHe}$ & $\mathrm{MsMgTr}$ & $\mathrm{XMs}$ & $\mathrm{StSMnPr}$ & - & + & - \\
\hline R. polyphyllus W.K. & $\mathrm{HKr}$ & $\mathrm{MgT}$ & $\mathrm{ScHe}$ & MsTr & Hy & PalAq & RLD & + & - \\
\hline R. repens $\mathrm{L}$. & $\mathrm{HKr}$ & MgT & $\mathrm{ScHe}$ & $\operatorname{MgTr}$ & HgMs & PalPr & - & + & + \\
\hline R. sceleratus L. & $\mathrm{T}$ & MsT & $\mathrm{ScHe}$ & $\mathrm{MsMgTr}$ & HgMs & PrPal & - & + & + \\
\hline Thalictrum flavum $\mathrm{L}$. & $\mathrm{HKr}$ & MgT & $\mathrm{ScHe}$ & MsMgTr & $\mathrm{Hg}$ & PalPr & - & + & + \\
\hline Th. lucidum $\mathrm{L}$. & $\mathrm{HKr}$ & MsT & $\mathrm{ScHe}$ & $\mathrm{MgTr}$ & $\mathrm{Hg}$ & $\operatorname{Pr}$ & RLD & + & + \\
\hline Th. simplex L. & $\mathrm{HKr}$ & MsT & $\mathrm{ScHe}$ & $\mathrm{MsMgTr}$ & Ms & $\mathrm{SMnPr}$ & - & + & + \\
\hline \multicolumn{10}{|l|}{ Resedaceae } \\
\hline Reseda lutea L. & $\mathrm{T}$ & $\mathrm{MgT}$ & $\mathrm{He}$ & MsTr & Ms & RuPtSt & Adv & + & + \\
\hline \multicolumn{10}{|l|}{ Rhamnacaceae } \\
\hline Frangula alnus Mill. & $\mathrm{nPh}$ & MgT & $\mathrm{HeSc}$ & Og-MgTr & HgMs & SMnSil & - & + & - \\
\hline Rhammus catartica $\mathrm{L}$. & $\mathrm{nPh}$ & MsT & $\mathrm{ScHe}$ & $\operatorname{MgMsTr}$ & XMs & SilSMn & - & + & - \\
\hline \multicolumn{10}{|l|}{ Rosaceae } \\
\hline Agrimonia eupatoria L. & $\mathrm{HKr}$ & MgT & $\mathrm{ScHe}$ & MgMsTr & XMs & SMnSt & - & + & + \\
\hline Armeniaca vulgaris Lam. & $\mathrm{Ph}$ & MgT & $\mathrm{He}$ & OgMsTr & $\mathrm{MsX}$ & RuSilCu & Adv & + & + \\
\hline Crataegus fallacina Klokov & $\mathrm{nPh}$ & MsT & $\mathrm{ScHe}$ & MsMsTr & MsX & SilSMnPtSt & - & + & - \\
\hline C. leimonogina Klokov & $\mathrm{Ph}$ & MsT & $\mathrm{ScHe}$ & MsTr & MsX & SilStSMn & - & + & + \\
\hline Filipendula ulmaria (L.) Maxim. & $\mathrm{G}$ & MgT & $\mathrm{HeSc}$ & $\operatorname{MgTr}$ & $\mathrm{MsHg}$ & SMnPalPr & - & + & - \\
\hline Fragaria campestris Steven & $\mathrm{HKr}$ & MsT & $\mathrm{ScHe}$ & MsTr & $\mathrm{XMs}$ & SMnSt & - & + & + \\
\hline F. vesca $\mathrm{L}$. & $\mathrm{HKr}$ & $\mathrm{MgT}$ & $\mathrm{HeSc}$ & $\operatorname{MgTr}$ & Ms & Sil & RLD & + & - \\
\hline F. viridis Duch. & $\mathrm{HKr}$ & MgT & $\mathrm{ScHe}$ & $\mathrm{MsTr}$ & $\mathrm{XMs}$ & StSMn & - & + & + \\
\hline Geum urbanum $\mathrm{L}$. & $\mathrm{HKr}$ & MsT & $\mathrm{ScHe}$ & OgMsTr & Ms & RuSil & - & + & - \\
\hline Malus praecox (Pall.) Borkh. & $\mathrm{Ph}$ & MsT & $\mathrm{HeSc}$ & MgMsTr & $\mathrm{XMs}$ & SilStSMn & - & + & + \\
\hline M. sylvestris Mill. & $\mathrm{Ph}$ & MgT & $\mathrm{HeSc}$ & MsTr & $\mathrm{XMs}$ & SMnSil & - & + & - \\
\hline Potentilla anserina $\mathrm{L}$. & $\mathrm{HKr}$ & EuT & $\mathrm{He}$ & AlkMgTr & $\mathrm{MsHg}$ & $\operatorname{Pr}$ & - & + & + \\
\hline P. argentea $\mathrm{L}$. & $\mathrm{HKr}$ & MsT & $\mathrm{He}$ & MsTr & MsX & SilPrStRu & - & + & + \\
\hline P. heptaphylla L. & $\mathrm{HKr}$ & MsT & $\mathrm{He}$ & MsTr & $\mathrm{MsX}$ & PrSil & RLD & + & + \\
\hline P. neglecta Baumg. & $\mathrm{HKr}$ & MgT & $\mathrm{He}$ & MsTr & MsX & RuSt & - & + & + \\
\hline P. obscura Willd. & $\mathrm{HKr}$ & MsT & $\mathrm{He}$ & $\operatorname{MgTr}$ & $\mathrm{XMs}$ & SilPt & - & + & + \\
\hline P. reptans $\mathrm{L}$. & $\mathrm{HKr}$ & $\mathrm{MgT}$ & $\mathrm{He}$ & MsTr & $\mathrm{HgMs}$ & $\mathrm{SMnPr}$ & - & + & + \\
\hline P. supina $\mathrm{L}$. & $\mathrm{THKr}$ & MT & $\mathrm{He}$ & $\mathrm{MsMgTr}$ & Ms & PrRu & - & - & + \\
\hline Prumus stepposa Kotov & $\mathrm{Ph}$ & MsT & $\mathrm{ScHe}$ & MsTr & MsX & SMnSt & - & + & + \\
\hline Pyrus communis L. & $\mathrm{Ph}$ & MgT & $\mathrm{ScHe}$ & MgMsTr & MsX & StSMnSil & - & + & + \\
\hline Rosa canina $\mathrm{L}$ & $\mathrm{nPh}$ & MgT & $\mathrm{ScHe}$ & $\mathrm{MsTr}$ & $\mathrm{XMs}$ & RuSMnSt & - & + & + \\
\hline R. corymbifera Borkh. & $\mathrm{nPh}$ & MgT & $\mathrm{ScHe}$ & MsTr & $\mathrm{MsX}$ & RuSMnSt & - & + & + \\
\hline Rubus caesius $\mathrm{L}$. & $\mathrm{nPh}$ & MgT & $\mathrm{ScHe}$ & MsT & Ms & RuSil & - & + & + \\
\hline Sanguisorba officinalis L. & $\mathrm{HKr}$ & MsT & $\mathrm{ScHe}$ & $\operatorname{MgTr}$ & Ms & $\mathrm{SMnPr}$ & RLD & + & + \\
\hline Rubiaceae & & & & & & & & & \\
\hline Galium aparine L. & $\mathrm{T}$ & $\mathrm{MgT}$ & $\mathrm{ScHe}$ & $\operatorname{MgTr}$ & $\mathrm{XMs}$ & SilRu & - & + & - \\
\hline G. boreale L. & $\mathrm{HKr}$ & MgT & $\mathrm{ScHe}$ & $\operatorname{MgTr}$ & Ms & PrSMn & - & + & - \\
\hline G. elongatum C.Presl. & $\mathrm{HKr}$ & MgT & $\mathrm{HeSc}$ & $\operatorname{MgTr}$ & $\mathrm{Hg}$ & SilPal & - & + & - \\
\hline G. mollugo L. & $\mathrm{HKr}$ & MsT & $\mathrm{ScHe}$ & MsTr & $\mathrm{XMs}$ & SilPr & - & + & - \\
\hline G. palustre L. & $\mathrm{HKr}$ & MsT & $\mathrm{ScHe}$ & $\operatorname{MgTr}$ & $\mathrm{MsHg}$ & PalPr & - & + & - \\
\hline G. physocarpum Ledeb. & $\mathrm{HKr}$ & $\mathrm{MgT}$ & $\mathrm{HeSc}$ & $\mathrm{MgTr}$ & Ms & SilPr & - & + & + \\
\hline G. rivale (Sibth.et Smim) Griseb. & $\mathrm{HKr}$ & $\mathrm{MgT}$ & $\mathrm{HeSc}$ & $\mathrm{MsTr}$ & $\mathrm{HgMs}$ & $\mathrm{SMnPr}$ & - & + & - \\
\hline G. rubioides L. & $\mathrm{HKr}$ & MgT & $\mathrm{He}$ & $\operatorname{MsTr}$ & Ms & $\operatorname{Pr}$ & - & + & + \\
\hline G. trifidum L. & $\mathrm{HKr}$ & MsT & $\mathrm{ScHe}$ & $\operatorname{MgTr}$ & $\mathrm{MsHg}$ & PrPal & RLD & + & + \\
\hline G. tyraicum Klokov & $\mathrm{HKr}$ & MsT & $\mathrm{HeSc}$ & $\mathrm{CaOgTr}$ & X & PtSilPs & RLD & + & - \\
\hline G. uliginosum $\mathrm{L}$. & $\mathrm{HKr}$ & MsT & $\mathrm{HeSc}$ & MsTr & $\mathrm{MsHg}$ & PrSilPal & RLD & + & - \\
\hline G. vaillantii DC. & $\mathrm{T}$ & MsT & $\mathrm{He}$ & MsTr & MsX & $\mathrm{StRu}$ & Adv & + & - \\
\hline Rubia tatarica (Trev.) Fr. Schmidt & $\mathrm{HKr}$ & MsT & $\mathrm{HeSc}$ & $\operatorname{MsTr}$ & MsX & PsPr & RLD & + & + \\
\hline Salicaceae & & & & & & & & & \\
\hline Populus alba L. & $\mathrm{Ph}$ & $\mathrm{MgT}$ & $\mathrm{He}$ & OgMsTr & $\mathrm{HgMs}$ & Sil & - & + & + \\
\hline
\end{tabular}




\begin{tabular}{|c|c|c|c|c|c|c|c|c|c|}
\hline 1 & 2 & 3 & 4 & 5 & 6 & 7 & 8 & 9 & 10 \\
\hline P. nigra L. & $\mathrm{Ph}$ & MgT & $\mathrm{He}$ & OgMsTr & HgMs & Sil & - & + & + \\
\hline P. tremula L. & $\mathrm{Ph}$ & MsT & $\mathrm{ScHe}$ & OgMsTr & $\mathrm{HgMs}$ & Sil & - & + & - \\
\hline Salix alba L. & $\mathrm{Ph}$ & MsT & $\mathrm{ScHe}$ & Og-MgTr & HgMs & Sil & - & + & + \\
\hline S. aurita L. & $\mathrm{Ph}$ & MsT & $\mathrm{ScHe}$ & Og-MgTr & $\mathrm{MsHg}$ & PsSMnPal & RLD & + & - \\
\hline S. caprea L. & $\mathrm{Ph}$ & $\mathrm{MgT}$ & $\mathrm{ScHe}$ & OgMsTr & Ms & SilSMn & RLD & + & - \\
\hline S. cinerea $\mathrm{L}$. & $\mathrm{Ph}$ & MgT & $\mathrm{ScHe}$ & MgMsTr & $\mathrm{MsHg}$ & SilPal & - & + & + \\
\hline S. fragilis L. & $\mathrm{Ph}$ & $\mathrm{MgT}$ & $\mathrm{He}$ & MsTr & $\mathrm{XMs}-\mathrm{MsHg}$ & SilPr & $\mathrm{Adv}$ & + & + \\
\hline S. pentandra L. & $\mathrm{Ph}$ & MgT & $\mathrm{HeSc}$ & MsTr & $\mathrm{MsHg}$ & PalSil & - & + & - \\
\hline S. rosmarinifolia $\mathrm{L}$. & $\mathrm{nPh}$ & MgT & $\mathrm{ScHe}$ & $\mathrm{MsTr}$ & MsX-MsHg & SilPs & - & + & - \\
\hline S. triandra L. & $\mathrm{Ph}$ & MsT & $\mathrm{He}$ & $\operatorname{MgTr}$ & $\mathrm{HgMs}$ & SilPr & - & + & + \\
\hline $\begin{array}{l}\text { S. vinogradovii A. Skvorts. } \\
\text { Scrophulariaceae }\end{array}$ & $\mathrm{Ph}$ & $\operatorname{MgT}$ & $\mathrm{He}$ & OgMsTr & $\mathrm{HgMs}$ & SilPr & - & + & + \\
\hline Euphrasia brevipila Burn. et Gremlin & $\mathrm{T}$ & MsT & $\mathrm{He}$ & MsTr & $\mathrm{XMs}$ & $\operatorname{Pr}$ & - & - & + \\
\hline E. stricta D.Wolff ex J.F.Lehm. & $\mathrm{T}$ & $\mathrm{MgT}$ & $\mathrm{HeSc}$ & OgMsTr & Ms & SMnSil & - & + & - \\
\hline Gratiola officinalis L. & $\mathrm{HKr}$ & MsT & $\mathrm{ScHe}$ & MsTr & Ms & PsPr & - & + & + \\
\hline Linaria genistifolia $(\mathrm{L}$.$) Mill$ & $\mathrm{HKr}$ & MgT & $\mathrm{He}$ & OgMsTr & $\mathrm{X}$ & PtStPs & - & + & - \\
\hline L. vulgaris Mill. & $\mathrm{G}$ & MsT & $\mathrm{ScHe}$ & MsTr & MsX & SMnPrRu & - & + & + \\
\hline Melampyrum nemorosum $\mathrm{L}$. & $\mathrm{T}$ & $\mathrm{MgT}$ & $\mathrm{ScHe}$ & OgMsTr & Ms & PsPrSil & RLD & + & - \\
\hline M. pratense $\mathrm{L}$. & $\mathrm{T}$ & MsT & $\mathrm{ScHe}$ & MsTr & Ms & PrSMnSil & RLD & + & - \\
\hline Odontines vulgaris Moench & $\mathrm{T}$ & $\mathrm{MgT}$ & $\mathrm{ScHe}$ & $\mathrm{Ms} T r$ & MsX & RuStPr & - & - & + \\
\hline Pedicularis dasystachys Schrenk & $\mathrm{HKr}$ & MgT & $\mathrm{He}$ & AlkMsTr & Ms & $\mathrm{HalPr}$ & RLD & - & + \\
\hline Rhinanthus vernalis (N.Zing.) Schischk.et Serg. & $\mathrm{T}$ & MgT & $\mathrm{He}$ & MsTr & Ms & RuSMnPr & - & + & - \\
\hline Scrophularia nodosa $\mathrm{L}$. & $\mathrm{HKr}$ & MsT & $\mathrm{HeSc}$ & MsMgTr & Ms & PrSMnSil & - & + & - \\
\hline Verbascum densiflorum Bertol. & $\mathrm{HKr}$ & $\mathrm{MgT}$ & $\mathrm{He}$ & MsMgTr & XMs & RuPsSMn & - & + & + \\
\hline V. lychnitis L. & $\mathrm{HKr}$ & MgT & $\mathrm{ScHe}$ & OgMsTr & MsX & RuSMn & - & + & - \\
\hline V. nigrum $\mathrm{L}$. & $\mathrm{HKr}$ & MgT & $\mathrm{ScHe}$ & MsTr & MsX & RuSMn & RLD & + & - \\
\hline Veronica anagallis-aquatica $\mathrm{L}$. & $\mathrm{HKr}$ & $\mathrm{MgT}$ & $\mathrm{He}$ & MsTr & $\mathrm{HelHg}$ & AqPal & - & + & + \\
\hline$V$. arvensis L. & $\mathrm{THKr}$ & MgT & $\mathrm{He}$ & MsTr & MsX & $\mathrm{StRu}$ & Adv & - & + \\
\hline V. austriaca $\mathrm{L}$. & $\mathrm{HKr}$ & MgT & $\mathrm{ScHe}$ & CaMsTr & MsX & SilSMnSt & - & + & - \\
\hline V. chamaedrys $\mathrm{L}$. & $\mathrm{HKr}$ & MgT & $\mathrm{ScHe}$ & $\mathrm{MsTr}$ & Ms & PrSMn & - & + & - \\
\hline$V$. hederifolia $\mathrm{L}$. & $\mathrm{T}$ & MgT & $\mathrm{ScHe}$ & MsTr & $\mathrm{XMs}$ & RuSMnSt & - & + & - \\
\hline V. longifolia $\mathrm{L}$. & $\mathrm{HKr}$ & MgT & $\mathrm{ScHe}$ & $\operatorname{MgTr}$ & $\mathrm{HgMs}$ & SilPr & - & + & + \\
\hline V. prostrata $\mathrm{L}$. & $\mathrm{HKr}$ & MgT & $\mathrm{ScHe}$ & AlkMsTr & MsX & StPrSMn & - & - & + \\
\hline V. scutellata L. & $\mathrm{HKr}$ & MsT & $\mathrm{He}$ & MsTr & $\mathrm{MsHg}$ & PalPr & RLD & + & + \\
\hline V. serpyllifolia $\mathrm{L}$. & $\mathrm{HKr}$ & $\mathrm{MgT}$ & $\mathrm{He}$ & MsTr & $\mathrm{HgMs}$ & RuSMnPr & RLD & + & + \\
\hline V. spicata L. & $\mathrm{HKr}$ & $\mathrm{MgT}$ & $\mathrm{ScHe}$ & MsTr & MsX & SMnSt & - & - & + \\
\hline V. spuria $\mathrm{L}$. & $\mathrm{HKr}$ & MgT & $\mathrm{ScHe}$ & MsTr & $\mathrm{XMs}$ & StSMn & - & + & - \\
\hline V. teucrium $\mathrm{L}$. & $\mathrm{HKr}$ & MgT & $\mathrm{ScHe}$ & OgMsTr & $\mathrm{XMs}$ & StSMn & - & + & + \\
\hline \multicolumn{10}{|l|}{ Solanaceae } \\
\hline Hyoscyamus niger L. & $\mathrm{HKr}$ & MT & $\mathrm{He}$ & $\mathrm{MsTr}$ & MsX & $\mathrm{Ru}$ & Adv & - & + \\
\hline Solanum dulcamara $\mathrm{L}$. & $\mathrm{Ch}$ & MsT & $\mathrm{ScHe}$ & OgMsTr & $\mathrm{MsHg}$ & SilPal & - & + & + \\
\hline S. nigrum L. & $\mathrm{T}$ & MgT & $\mathrm{He}$ & MsTr & $\mathrm{Ms}$ & $\mathrm{Ru}$ & Adv & + & + \\
\hline \multicolumn{10}{|l|}{ Tiliaceae } \\
\hline \multicolumn{9}{|l|}{ Ulmaceae } & - \\
\hline Ulmus glabra Huds. & $\mathrm{Ph}$ & $\operatorname{MgT}$ & $\mathrm{HeSc}$ & $\operatorname{MgTr}$ & Ms & Sil & - & + & - \\
\hline U. laevis Pall. & $\mathrm{Ph}$ & MsT & $\mathrm{HeSc}$ & Og-MgTr & Ms & Sil & - & + & - \\
\hline U. minor Mill. & $\mathrm{Ph}$ & $\mathrm{MgT}$ & $\mathrm{ScHe}$ & MsTr & MsX & SilSMn & - & + & + \\
\hline U. pumila L. & $\mathrm{Ph}$ & MgT & $\mathrm{ScHe}$ & OgMsTr & MsX & SilCuRu & Adv & + & + \\
\hline U. suberosa Moench & $\mathrm{Ph}$ & MgT & $\mathrm{ScHe}$ & MsTr & $\mathrm{X}$ & SMn & - & + & + \\
\hline \multicolumn{10}{|l|}{ Urticaceae } \\
\hline Urtica diocica $\mathrm{L}$. & G & $\operatorname{MgT}$ & $\mathrm{He}-\mathrm{Sc}$ & $\operatorname{MsMgTr}$ & Ms & SilRu & - & + & + \\
\hline \multicolumn{10}{|l|}{ Valerianaceae } \\
\hline Valeriana officinalis L. & $\mathrm{HKr}$ & MsT & $\mathrm{ScHe}$ & $\operatorname{MgTr}$ & $\mathrm{HgMs}$ & $\mathrm{SMnPr}$ & RLD & + & - \\
\hline V. rossica P. Smirn. & $\mathrm{HKr}$ & MsT & $\mathrm{ScHe}$ & $\operatorname{MgTr}$ & MsX & PrSt & RLD & + & + \\
\hline V. stolonifera Czern. & $\mathrm{HKr}$ & MsT & $\mathrm{ScHe}$ & $\operatorname{MsMgTr}$ & Ms & PrSMnSil & RLD & + & - \\
\hline \multicolumn{10}{|l|}{ Verbenaceae } \\
\hline Verbena officinalis L. & $\mathrm{HKr}$ & $\operatorname{MgT}$ & $\mathrm{ScHe}$ & MsTr & Ms & $\mathrm{SMnPrRu}$ & Adv & + & + \\
\hline Viola canina L. & $\mathrm{HKr}$ & MgT & $\mathrm{ScHe}$ & MsTr & Ms & PrSil & - & + & - \\
\hline V. collina Besser & $\mathrm{HKr}$ & MsT & $\mathrm{ScHe}$ & MsTr & MsX & SMnSt & - & + & + \\
\hline V. hirta L. & $\mathrm{HKr}$ & MsT & $\mathrm{ScHe}$ & $\mathrm{MsMgTr}$ & $\mathrm{XMs}$ & StSil & - & + & + \\
\hline V. matutina Klokov & $\mathrm{THKr}$ & MsT & $\mathrm{ScHe}$ & MsTr & XMs & RuSMnPr & - & + & - \\
\hline V. mirabilis L. & $\mathrm{HKr}$ & MsT & $\mathrm{HeSc}$ & $\mathrm{MsMgTr}$ & Ms & Sil & - & + & - \\
\hline V. odorata $\mathrm{L}$. & $\mathrm{HKr}$ & MgT & $\mathrm{HeSc}$ & MsMgTr & Ms & RuSil & - & + & + \\
\hline V. suavis M.Bieb. & $\mathrm{HKr}$ & MgT & $\mathrm{HeSc}$ & MsTr & HgMs & PrSil & - & + & - \\
\hline V.tricolor L. & $\mathrm{THKr}$ & MsT & $\mathrm{ScHe}$ & MsTr & MsX & RuSMnPr & - & + & - \\
\hline
\end{tabular}

Notes: Climamorphs: $\mathrm{Ph}$ - phanerophytes, HKr - hemicryptophytes, G-geophytes, T - therophytes, $\mathrm{Ch}$ - hamephytes, Hel - helophytes, Hd - hydatophytes. Thermomorphs: MT - microtherms (types of northern climatic zones), MsT - mesotherms (types of temperate climatic zone), MgT - megatherms (species of subtropical or tropical climatic zones), EuT - eurytherms (species that can grow in almost all climatic zones). Heliomorphs: He (Heliophyton) - heliophytes (obligate sun-loving plants), Sc (Sciophyton) sciophytes (obligate shade-loving plant species). Trophomorphs: OgTr (Oligotroph) - oligotrophs (species growing on nutrient-poor soils), MsTr (Mesotroph) - mesotrophs (species growing on soils medium in fertility), MgTr (Megatroph) - megatrophs (species tending to prefer soil high in fertility), Alk (Alkotroph) - alkotrophs (species growing on saline soils), Par - parasite. Hygromorphs: Hy (Hydatophyton) - hydatophytes (underwater plants, mostly totally immersed in water), Pl (Pleistophyton) - pleistophytes (species floating on the water's surface), Hel (Helophyton) - helophytes (species of shallow-water habitats), Hg (Hygrophyton) - hygrophytes (species of wet soils), Ms (Mesophyton) mesophytes (inhabitants of fresh soils), X (Xerophyton) - xerophytes (species of dry habitats). Cenomorphs: Aq (Aqant) - aquant (aquatic species), Pal (Paludosus) - paludant (swampy species), Pr (Pratensis) - pratant (meadow species), Sil (Silvaticus) - sylvant (forest species), St (Stepposus) - stepant (steppe species), SMn (Margosilvaticus) - sylvomargoant (forest margin species), Ps (Psammophyton) - psammophyte (species of sandy soils), Pt (Petrophyton) - petrophyte (species of stony biotopes), Ru (Ruderatus) ruderant (ruderal species), Hal (Halophyton) - halophyte (species of saline soils), $\mathrm{Cu}$ (Cultus) - culturant (cultural species). Adventive status - Adv. The rare flora fraction: RLD - Red List of Dnipropetrovsk Region; RBU - Red Book of Ukraine; ERL - European Red List. 
Table 3

Comparative analysis bioecological phytodiversity of the Prysamar'ya forest ecosystems

\begin{tabular}{|c|c|c|c|}
\hline Ecomorphs & $\begin{array}{l}\text { Floodplain } \\
\text { forests }\end{array}$ & $\begin{array}{c}\text { Territory of } \\
\text { treeless floodplain }\end{array}$ & $\begin{array}{c}\text { Floodplain } \\
\text { territory }\end{array}$ \\
\hline \multicolumn{4}{|c|}{ The number of types of different climamorphs } \\
\hline Therophytes & 102 & 99 & 124 \\
\hline Geophytes & 72 & 55 & 88 \\
\hline Hemicriptophytes & 377 & 285 & 439 \\
\hline Hamephytes & 7 & 7 & 10 \\
\hline Phanerophytes & 57 & 29 & 56 \\
\hline Helophytes & 7 & 6 & 7 \\
\hline Hydatophytes & 4 & 4 & 4 \\
\hline \multicolumn{4}{|c|}{ The number of types of different thermomorphs } \\
\hline Microtherms & 12 & 12 & 16 \\
\hline Mesotherms & 248 & 187 & 284 \\
\hline Megatherms & 337 & 260 & 395 \\
\hline Eurytherms & 31 & 27 & 32 \\
\hline \multicolumn{4}{|c|}{ The number of types of different heliomorphs } \\
\hline Sciophytes & 15 & 1 & 15 \\
\hline Heliosciophytes & 112 & 29 & 115 \\
\hline Scioheliophytes & 283 & 193 & 310 \\
\hline Heliophytes & 216 & 262 & 288 \\
\hline \multicolumn{4}{|c|}{ The number of types of different trophomorphs } \\
\hline Megatrophs & 179 & 70 & 203 \\
\hline Mesotrophs & 401 & 353 & 460 \\
\hline Oligotrophs & 37 & 42 & 44 \\
\hline Alkotrophs & 6 & 17 & 18 \\
\hline Parasites & 3 & 3 & 3 \\
\hline \multicolumn{4}{|c|}{ The number of types of different hygromorphs } \\
\hline Xerophytes & 91 & 121 & 139 \\
\hline Mesophytes & 355 & 241 & 418 \\
\hline Hygrophytes & 127 & 87 & 129 \\
\hline Helophytes & 14 & 14 & 14 \\
\hline Hydatophytes & 28 & 15 & 28 \\
\hline Pleistophytes & 10 & 7 & 10 \\
\hline \multicolumn{4}{|c|}{ The number of types of different cenomorphs } \\
\hline Stepant & 55 & 66 & 71 \\
\hline Sylvant & 150 & 32 & 154 \\
\hline Sylvomargoant & 35 & 20 & 35 \\
\hline Psammophyte & 16 & 16 & 23 \\
\hline Petrophyte & 3 & 4 & 7 \\
\hline Pratant & 151 & 151 & 198 \\
\hline Paludant & 73 & 47 & 64 \\
\hline Aquant & 50 & 36 & 52 \\
\hline Ruderant & 90 & 103 & 114 \\
\hline Halophyte & 3 & 10 & 10 \\
\hline \multicolumn{4}{|c|}{ The rare flora fraction } \\
\hline European Red List & 1 & 2 & 2 \\
\hline Red Book of Ukraine, 2009 & 20 & 14 & 24 \\
\hline $\begin{array}{l}\text { Red List of Dnipropetrovsk } \\
\text { Region, } 2010\end{array}$ & 134 & 60 & 148 \\
\hline Adventive* & 89 & 97 & 105 \\
\hline Total species & 628 & 486 & 728 \\
\hline
\end{tabular}

Note: *-amendments of Protopopova (1991).

The bioecomorphic composition of flora is dominated by: hemicryptophytes (439), megatherms (395 species), scioheliophytes (310), mesophytes (418), mesotrophs (460), and cenomorphs dominated by forest (154) and meadow (198) species (Table 3).

\section{Discussion}

The analysis of floral richness shows that the floodplain forests of the Samara contain 628 species of vascular plants from 728 species of the entire floodplain territory and 887 species of the entire flora of the Samara River area. This confirms the position that the floodplains of the valley landscapes of the temperate zone of Europe (Schindler et al., 1916; Filippová \& Pohanka, 2019) have the highest level of biodiversity.

A significant number of ruderal species (16.2\%), of which $92.4 \%$ are adventive, evidence anthropogenic transformation of the Samara floodplain flora. Of the floodplain landscape flora, 628 species of vascular plants grow in forest communities, and 486 species grow in transformed, treeless floodplain territories (Table 3).
Of the total flora composition in the floodplain landscape of the Samara River area, within the spectrum of biomorphs and climamorphs there are almost twice as many wood and shrubby species as in treeless areas, and the remaining categories increased by approximately one third. Among helimorphs, there were several times more sciophytes in the forests, among the hygromorphs and there were a third more hygrophytes and mesophytes, and among trophomorphs there were twice as many megatrophs.

Ruderalization of the flora is more typical for the treeless floodplain areas (103 species) compared to the wooded floodplain (90 species).

\section{Conclusion}

Despite the long-term anthropogenic transformation, the valley-terrace landscape territory of the Samara River area, as one of the reference native complexes of the subarid territory of Eastern Europe, has preserved a significant level of phytodiversity. In this regard, it is planned to develop a National Park "Samara Bor" within this area.

The floodplain landscape is the richest in species and most diverse part of this complex. Its flora includes 728 plant species (including 132 rare ones), of which 631 grow in the forest communities, and 487 in anthropogenically transformed, treeless floodplain areas.

In the forest flora, the number of tree and shrubby species, scyophytes, hygrophytes, and megatrophs significantly increases, and the number of ruderal plant species decreases.

The floristic composition of the floodplain forests of the middle river of the subarid region is much richer and more diverse than the flora of the treeless floodplain areas, and this should encourage measures for their protection and restoration. Afforestation of floodplain territories within the steppe zone of Ukraine should be a priority in comparison with other landscapes.

\section{References}

Akinfiyev, I. Y. (1908). Botanicheskiy ocherk Novomoskovskogo uyezda [Botanical sketch of the Novomoskovsky district]. Materialy po otsenke zemel' Yekaterinoslavskoy gubernii, Yekaterinoslav, 1-15 (in Russian).

Alekseyev, Y. Y., Bel'gard, A. L., Gubanov, I. A., Kovaleva, O. V., Tarasov, V. V., \& Travleyev, A. P. (1986). Rastitel'nyy i pochvennyy pokrov Prisamar'ya Dneprovskogo [Vegetation and soil cover of the Prisamarya of Dnieper]. Izdatel'stvo DGU, Dnepropetrovsk (in Russian).

Baranovski, B. O., \& Tarasov, V. V. (2010). Chervona knyha Dnipropetrovskoyi oblasti (Roslynnyy svit) [Red Book of Dnipropetrovsk region (Flora)]. Dnipropetrovsk (in Ukrainian).

Baranovsky, B. A. (2000). Rastitel'nost' ruslovogo ravninnogo vodohranilishha [Vegetation of the channel of a lowland reservoir]. Izdatel'stvo DNU, Dnepropetrovsk (in Russian).

Baranovsky, B. A., Manjuk, V. V., Ivanko, I. A., \& Karmyzova, L. A. (2017). Analiz flory nacional'nogo pryrodnogo parku "Oril's'kij" [Analysis of the flora of the Orilsky National Park]. Lira, Dnipro (in Ukrainian).

Baranovsky, B. A. (2016). Chuzhoridni vydy sudynnykh roslyn natsionalnoho parku "Samarskyy Bir" [Alien species of vascular plants of Samara Bir National Park]. Ecology and Noospherology, 27(3-4), 16-26 (in Ukrainian).

Baranovsky, B. A. (2017). Vydelenie novoj cenomorfy v kontekste razvitija sistemy ekomorf A. L. Bel'garda [Differentiation of new coenomorph in context of the Belgard's ecomorph system development]. Ecology and Noospherology, 28(3-4), 16-26 (in Russian).

Bel'gard, A. L. (1950). Lesnaja rastitel'nost' jugo-vostoka USSR [Forest vegetation of southeast Ukrainian SSR]. KGU, Kiev (in Russian).

Biurrun, I., Campos, J. A., García Mijangos, I., Herrera, M., \& Loidi, J. (2016). Floodplain forests of the Iberian Peninsula: Vegetation classification and climatic features. Applied Vegetation Science, 19, 336-354.

Brygadyrenko, V. V. (2015). Community structure of litter invertebrates of forest belt ecosystems in the Ukrainian steppe zone. International Journal of Environmental Research, 9(4), 1183-1192.

Brygadyrenko, V. V. (2016). Evaluation of ecological niches of abundant species of Poecilus and Pterostichus (Coleoptera: Carabidae) in forests of the steppe zone of Ukraine. Entomologica Fennica, 27(2), 81-100.

Bussotti, F., Pollastrini, M., Holland, V., \& Bruggeman, W. (2015). Functional traits and adaptive capacity of European forests to climate change. Environmental and Experimental Botany. 111(3), 91-113.

Colangelo, M., Camarero, J. J., Ripullone, F., Gazol, A., Sánchez-Salguero, R., Oliva, J., \& Redondo, M. A. (2018). Drought decreases growth and increases 
mortality of coexisting native and introduced tree species in a temperate floodplain forest. Forests, 9(4), 205.

Elenevskij, A. G. (1978). Sistematika i geografiya veronik SSSR i prilezhashhikh stran [Systematics and geography of Veronica of the USSR and neighboring countries]. Nauka, Moscow (in Russian).

Filippovová, J., \& Pohanka, T. (2019). Environmental assessment of central European floodplain forests: A case study from the Morava River alluvium. Polish Journal of Environmental Studies, 28(6), 4511-4517.

Gorb, A. S., \& Duk, N. M. (2006). Klimat Dnepropetrovskoy oblasti [Climate of the Dnipropetrovsk region]. Izdatel'stvo DNU, Dnepropetrovsk (in Russian).

Grytsan, Y. I. (2000). Ekolohichni osnovy peretvoryuyuchoho vplyvu lisovoyi roslynnosti na stepove seredovyshche [The ecological bases of transformative influence of forest vegetation on the steppe environment]. Izdatel'stvo DNU, Dnipropetrovsk (in Ukrainian).

Grytsan, Y. I., Karas, O. G., \& Baranovsky, B. O. (2005). Osoblyvosti meteorolohichnykh protsesiv zaplavy (na prykladi Samarskoho boru) [Features of meteorological processes of the floodplain (on the example of the Samara Forest)]. Visnyk Kryvorizkoho Tekhnichnoho Universytetu, 10, 222-227 (in Ukrainian).

Heklau, H., Jetschke, G., Bruelheide, H., Seidler, G., \& Haider, S. (2019). Species specific responses of wood growth to flooding and climate in floodplain forests in Central Germany. iForest, 12, 226-236.

Hossain, K., Yadav, S., Quaik, S., Pant, G., Maruthi, A. Y., \& Ismail, N. (2016). Vulnerabilities of macrophytes distribution due to climate change. Theoretical and Applied Climatology, 129, 1123-1132.

Koshelev, V. A., Pakhomov, O. Y., \& Busel, V. A. (2020). The formation of sclerophilic ornythocomplexes in the quarries in the south of Ukraine and their conservation prospects. Ecology, Environment and Conservation, 26(1), 411-419.

Lavergne, S., Mouquet, N., Thuiller, W., \& Ronce, O. (2010). Biodiversity and climate change: Integratin gevolutionary and ecological responses of species and communities. Annual Review of Ecology, Evolution and Systematics, 41, 321-350.

Mikac, S., Žmegač, A., Trlin, D., Paulić, V., Oršanić, M., \& Anić, I. (2018). Drought-induced shift in tree response to climate in floodplain forests of Southeastern Europe. Scientific Reports, 8, 16495.
Mosyakin, S. L., \& Fedoronchuk, M. M. (1999). Vascular plants of Ukraine (Nomenclatural checklist). Naukova Dumka, Kyiv.

Ortmann-Ajkai, A., Csicsek, G., Hollós, R., Magyaros, V., Wágner, L., \& Lóczy, D. (2018). Twenty-years' changes of wetland vegetation: Effects of floodplain-level threats. Wetlands, 38, 591-604.

Prokudin, J. N., \& Dobrochaeva, D. N. (Eds.). (1987). Opredelitel' vysshih rastenij Ukrainy [Identification guide to higher plants of Ukraine]. Naukova Dumka, Kiev (in Russian).

Protopopova, V. V. (1991). Sinantropnaya flora Ukrainy i puti yeyo razvitiya [Synanthropic flora of Ukraine and ways of its development]. Naukova Dumka, Kiev (in Russian).

Ramenskiy, L. G. (1971). Problemy i metody izuchenija rastitel'nogo pokrova [Problems and methods of studying vegetation]. Nauka, Leningrad (in Russian).

Roshchyna, N. O., \& Baranovski, B. O. (2019). Hidroloho-hidrobotanichna typolohiya ozer pivnichno-stepovoho Prydniprov'ya Hydrological and hydrobotanic typology of the lake of North-Steppe Dnieper region. Ecology and Noospherology, 30(2), 125-131 (in Ukrainian).

Schindler, S., O’Neill, F. H., Biró, V., Damm, C., Gasso, V., Kanka, R., Sluis, T., Krug, A., Lauwaars, S. G., Sebesvari, Z., Pusch, M., Baranovski, B., Ehlert, T., Neukirchen, B., Martin, J. R., Euller, K., Mauerhofer, V., \& Wrbka, T. (2016). Multifunctional floodplain management and biodiversity effects: A knowledge synthesis for six European countries. Biodiversity and Conservation 25, 1349-1382.

Stojanović, D. B., Levanič, T., Matović, B., \& Orlović, S. (2015). Growth decrease and mortality of oak floodplain forests as a response to change of water regime and climate. European Journal of Forest Research, 134, 555-567.

Tarasov, V. V. (2012). Flora Dnipropetrovs'koi' ta Zaporiz'koi' oblastej [Dnipropetrovsk and Zaporizhia regions flora]. Lira, Dnipropetrovsk (in Ukrainian).

Tarasov, V. V., Alekseyev, Y. A., \& Gubanov, I. A. (1988). Rastitel'nyye resursy Prisamar'ya Dneprovskogo [Plant resources of the Prisamarya of Dnieper]. Izdatel'stvo DGU, Dnepropetrovsk (in Russian).

Vysotskiy, G. N. (1950). Ucheniye o vliyanii lesa na izmeneniye sredy yego proizrastaniya i na okruzhayushcheye prostranstvo (ucheniye o lesnoy pertinentsii) [The doctrine of the influence of the forest on the change in the environment of its growth and on the surrounding space (the doctrine of forest continent)]. Goslesbumizdat, Moscw (in Russian). 